DEGREE PROJECT IN ELECTRICAL ENGINEERING, SECOND CYCLE, 30 CREDITS

STOCKHOLM, SWEDEN 2021

\title{
Generalized Talagrand Inequality for Sinkhorn Distance using Entropy Power Inequality
}

\author{
SHUCHAN WANG
}




\section{Generalized Talagrand Inequality for Sinkhorn Distance using Entropy Power Inequality}

SHUCHAN WANG

Master's Programme, Information and Network Engineering, 120 credits

Date: September 20, 2021

Supervisor: Photios A. Stavrou

Examiner: Mikael Skoglund

School of Electrical Engineering and Computer Science

Swedish title: Generaliserad Talagrand Inequality för Sinkhorn

Distance med Entropy Power Inequality 
(c) 2021 Shuchan Wang 


\section{Abstract}

Measure of distance between two probability distributions plays a fundamental role in statistics and machine learning. Optimal Transport (OT) theory provides such distance. Recent advance in OT theory is a generalization of classical OT with entropy regularized, called entropic OT. Despite its convenience in computation, it still lacks theoretical support. In this thesis, we study the connection between entropic OT and Entropy Power Inequality (EPI). First, we prove an HWI-type inequality making use of the infinitesimal displacement convexity of OT map. Second, we derive two Talagrand-type inequalities using the saturation of EPI that corresponds to a numerical term in our expression. We evaluate for a wide variety of distributions this term whereas for Gaussian and i.i.d. Cauchy distributions this term is found in explicit form. We show that our results extend previous results of Gaussian Talagrand inequality for Sinkhorn distance to the strongly log-concave case. Furthermore, we observe a dimensional measure concentration phenomenon using the new Talagrand-type inequality.

\section{Keywords}

Optimal Transport, Entropic Optimal Transport, Sinkhorn Distance, Talagrand Inequality, Entropy Power Inequality 
ii | Abstract 


\section{Sammanfattning}

Mått på avstånd mellan två sannolikhetsfördelningar spelar en grundläggande roll i statistik och maskininlärning. Optimal transport (OT) teori ger ett sådant avstånd. Nyligen framsteg inom OT-teorin är en generalisering av klassisk OT med entropi-reglerad, kallad entropisk OT. Trots dess bekvämlighet i beräkning saknar det fortfarande teoretiskt stöd. I denna avhandling studerar vi sambandet mellan entropisk OT och Entropy Power Inequality (EPI). Först bevisar vi en ojämlikhet av HWI-typ med användning av OT-kartans oändliga förskjutningskonvexitet. För det andra härleder vi två Talagrand-typkvaliteter med mättnaden av EPI som motsvarar ett numeriskt uttryck vårt uttryck. Vi utvärderar för ett brett utbud av distributioner den här termen för Gauss och i.i.d. Cauchy-distributioner denna term finns oförklarlig form. Vi visar att våra resultat utökar tidigare resultat av GaussianTalagrand-ojämlikhet för Sinkhorn-avstånd till det starkt log-konkava fallet. Dessutom observerar vi ett dimensionellt mått koncentrationsfenomen mot den nya Talagrand-typen ojämlikhet.

\section{Nyckelord}

Optimal Transport, Entropisk Optimal Transport, Sinkhorn Distance, Talagrand Inequality, Entropy Power Inequality 
iv | Sammanfattning 


\section{Acknowledgments}

First of all, I would like to thank my supervisor Dr. Photios A. Stavrou, who helped me a lot during my thesis. Photios always carefully reads the manuscript and gives me very detailed feedback efficiently. The ideas in this thesis are partly based on his constructive comments.

I would also like to express my gratitude to my examiner Prof. Mikael Skoglund for giving me the opportunity to do this thesis. His PhD course, Information Theory for Statistics and Learning, has also given me many insights for this thesis.

This thesis is supported in part by Karl Engvers Foundation and by KTH under One-Year Scholarship. Finally, I am thankful to my family, who support me during my studies in Sweden.

Stockholm, September 2021

Shuchan Wang 
vi|Acknowledgments 


\section{Contents}

1 Introduction 1

2 Background $\quad 3$

2.1 Optimal Transport . . . . . . . . . . . . . . 4

2.2 Entropic OT . . . . . . . . . . . . . . . . 7

2.2.1 Existence of solution to $\left(\mathcal{P}_{R}\right) \ldots \ldots \ldots \ldots$

2.2.2 Regularized OT . . . . . . . . . . . . . . 8

2.2.3 Sinkhorn Algorithm . . . . . . . . . . . . . 12

2.3 Time-dependent Monge-Kantorovich Problem . . . . . . . . . 14

2.4 Infinitesimal Displacement Convexity . . . . . . . . . . . 16

2.5 Talagrand Inequality . . . . . . . . . . . . . . . . . 18

2.6 Measure Concentration . . . . . . . . . . . . . . . . . . . 19

2.7 Conditional Sinkhorn Distance . . . . . . . . . . . . 21

2.8 Entropy Power Inequality and Deconvolution . . . . . . . 22

3 Main Results $\quad 25$

3.1 Theoretical Results . . . . . . . . . . . . . . . 25

3.2 Physical Interpretation of Our Results . . . . . . . . . . 30

3.3 Numerical Simulations . . . . . . . . . . . . . 31

4 Conclusions and Future work $\quad 37$

4.1 Conclusions . . . . . . . . . . . . . . . . 37

4.2 Future work . . . . . . . . . . . . . . 38

4.2.1 Entropy Bound . . . . . . . . . . . . . 38

4.2.2 Connections with Stochastic Process . . . . . . . . . 40

$\begin{array}{ll}\text { References } & 47\end{array}$

A More Proofs for Chapter $3 \quad 53$

A.1 Proof of Theorem $12 \ldots \ldots \ldots 53$ 
viii | Contents

A.2 Proof of Corollary $13.2 \ldots \ldots \ldots \ldots$

A.3 Proof of Theorem $14 \ldots \ldots \ldots \ldots$

A.4 Proof of Theorem $15 \ldots \ldots \ldots \ldots$

A.5 Useful Definitions, Theorems and Lemmas . . . . . . . . 57 


\section{List of Figures}

2.1 Color maps of the optimizer $P^{*}$ with respect to different $R$ for $\left(\mathcal{P}_{R}\right)$, or equivalently, different $\epsilon$ for $\left(\mathcal{P}_{\epsilon}\right)$. . . . . . . 10

2.2 Interpolations between two Gaussian distributions by different metrics. . . . . . . . . . . . . 16

3.1 Plot of the numerical term $\mathrm{C}$ subject to the information constraint $R$ evaluated with respect to different distributions for the one dimensional case. . . . . . . . . . . . . . . 28

3.2 Numerical simulations and bounds (3.2) subject to different $R$. 33

3.3 Numerical simulations and bounds (3.6) subject to different $R$. 34

3.4 Probability densities of $d P_{X}=e^{-V}, V=(x / 5)^{2} / 2+(x / 5)^{4}+$ $k, k \in \mathbb{R}$ and $d P_{Y} \sim \mathcal{N}\left(0, \frac{1}{25}\right) \ldots \ldots \ldots \ldots \ldots$

3.5 Numerical simulations and bounds subject to different $R$, for $d \mu=e^{-V}, V=(x / 5)^{2} / 2+(x / 5)^{4}+k, k \in \mathbb{R}$ and $d \mu \sim$

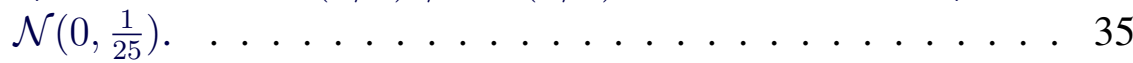

3.6 Numerical simulations and bounds subject to different $R$, for $d \mu=e^{-V}, V=(x / 5)^{2} / 2+(x / 5)^{8}+k, k \in \mathbb{R}$ and $d \mu \sim$

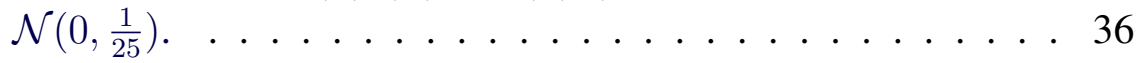

3.7 Sinkhorn distances between isotropic Gaussians on different dimensions. . . . . . . . . . . . . 36 
x | LIST OF FIGURES 


\section{List of acronyms and abbreviations}

DP Duality Problem

EPI Entropy Power Inequality

KL Kullback-Leibler

KP Kantorovich Problem

MP Monge Problem

OT Optimal Transport

PDE partial differential equation

SDE stochastic differential equation

\section{Notations}

Let $A$ and $B$ be two sets. The set subtraction is denoted by $A \backslash B$. For a set $A$, $1_{A}$ is its indicator function, i.e., $1_{A}(x)=1$ if $x \in A$, and 0 otherwise. $\bar{A}$ is the closure of a set $A$ in a metric space.

For two matrices $A$ and $B$ of the same dimension, $A \odot B$ denotes Hadamard product. $A^{\top}$ is matrix transpose. For two functions $f$ and $g, f \circ g$ denotes function composition.

$\mathbb{N}$ is the set of positive integers $\{1,2,3, \ldots\} . \mathbb{R}$ is the set of real numbers. $\mathbb{R}^{n}$ is the $n$-dimension Euclidean space. $\mathbb{R}_{+}$denotes the set $\left\{x \in \mathbb{R}_{+} ; x \geq 0\right\}$.

$\nabla$ is the gradient operator, $\nabla \cdot$ is the divergence operator, $\Delta$ is the Laplacian operator, $D^{2}$ is the Hessian operator, $I_{n}$ is the identity matrix, $I d$ is the identity map, $\|\cdot\|$ is the Euclidean norm, $C^{k}$ is the set of functions that k-times continuously differentiable.

Let $\mathcal{X}$ be a Polish space, i.e., a separable complete metric space. We denote $\mathcal{P}(\mathcal{X})$ as the set of all probability measures on $\mathcal{X}$. For a Polish space $\mathcal{X}$ with reference measure $\nu$, we denote $\mathcal{P}^{a c}(\mathcal{X})$ as the elements in $\mathcal{P}(\mathcal{X})$ which are absolutely continuous with respect to $\nu$.

Let $\mathcal{X}, \mathcal{Y}$ be two Polish spaces. Let $\mu$ be a Borel measure on $\mathcal{X}$. For a measurable map $T: \mathcal{X} \rightarrow \mathcal{Y}, T_{\#} \mu$ denotes pushing forward of $\mu$ to $\mathcal{Y}$, i.e. for 
xii | List of acronyms and abbreviations

all $A \subset \mathcal{Y}, T_{\#} \mu[A]=\mu\left[T^{-1}(A)\right]$. For $p \geq 1, L^{p}(\mathcal{X})$ or $L^{p}(d \mu)$ denotes the Lebesgue space of $p$-th order for the reference measure $\mu$.

We write a random vector $X$ on a Polish space $\mathcal{X}$ in capital letter, an element $x \in \mathcal{X}$ in lower-case letter. $h(\cdot), I(\cdot ; \cdot), D(\cdot \| \cdot), J(X), I(\cdot \mid \cdot)$ denote differential entropy, mutual information, KL-divergence, Fisher information and relative Fisher information, respectively.

$\mathrm{CD}(K, N)$ means 'curvature-dimension condition $(K, N)$ ', which means that the Ricci curvature is lower bounded by $K g$ ( $K$ a real number and $g$ the Riemannian metric) and dimension is upper bounded by $N(N$ a real number not less than 1).

All the logarithms are natural logarithms. $\exists$ ! is unique existence. $*$ is the convolution operator. 'R.H.S' is the abbreviation of 'Right Hand Side'. $\mathcal{O}$ is the big $\mathrm{O}$ notation. 


\section{Chapter 1}

\section{Introduction}

OT theory studies how to transport one measure to another in the path with minimal cost. Wasserstein distance is the cost given by the optimal path and closely connected with information measures [1, 2, 3, 4, 5].

During the last decade, OT has been studied and applied extensively, especially in machine learning community, see, e.g., [6, 7, 8, 9]. Entropic OT, a technique to approximate the solution of original OT, was given for computational efficiency in [10]. A key concept in entropic OT is Sinkhorn distance, which is a generalization of Wasserstein distance with entropic constraint.

One of the applications of OT is in the field of functional inequalities with geometrical content, which includes, for example, Talagrand inequality, HWI inequality, Brunn-Minkowski inequality, etc. Talagrand inequality in [1] upper bounds Wasserstein distance by Kullback-Leibler (KL) divergence. Recent results in $[2,4]$ obtain several refined Talagrand inequalities with dimensional improvement on multidimensional Eucledian space. These inequalities bound Wasserstein distance with entropy power, which is sharper compared to KLdivergence. Later, [11] shows the dimensional improvement can be used in entropic OT and gives a Gaussian Talagrand inequality for Sinkhorn distance. The Talagrand inequalities above can directly give results on measure concentration [5]. A strong data processing inequality is also obtained in [11] and yields a bound for the capacity of the relay channel.

In this thesis, we propose a new approach to study entropic OT using EPIs (for details on EPI see, e.g., [12, 13, 14]), which is to capture the uncertainty caused by entropic constraint by EPI. As a first contribution, we give an HWItype inequality for Sinkhorn distance by modifying Bolley's proof in [4] (see Theorem 12). As a second contribution, we derive two new Talagrand-type 
inequalities (see Theorems 13,14). These inequalities are obtained via a numerical term related to the saturation of EPI. The value of this term is explicit for Gaussian and i.i.d. Cauchy distributions. We also provide via numerical simulations the computation of this numerical term for a variety of distributions (see Remark 3.1). Moreover, we show that Theorem 13 coincides with a result by Bolley [4, Theorem 2.1] (see Corollary 13.1 and the discussion in Remark 3.1) and that Theorem 14 recovers the result by Bai et al. [11, Theorem 2.2] (see Remark 3.1). Furthermore, we show that Theorem 13 can give a dimensional measure concentration result in Corollary 13.2. Finally, we give a physical intuition of our results using McCann's interpolation. 


\section{Chapter 2}

\section{Background}

OT theory has appeared in many realistic problems, e.g., inverse problem [15], computer vision [16], control theory [17, 18, 19, 20, 21, 22], etc. Moreover, with the in-depth study of deep learning methods, people have also realized the foundational role played by the OT theory. Deep learning can be understood as learning the probability distribution on a manifold, so the transformation between probability distributions and measurement of the distance between probability measures are the core topics of deep learning. OT theory provides a theoretical basis and computational tools for solving these problems.

OT problem is known to be equivalent to Monge-Ampère equation. However, the equivalent partial differential equation (PDE) is highly nonlinear. It has always been challenging to accurately compute the OT map. Nowadays, in the engineering field, especially in the field of deep learning, people discretize the probability measure and add entropy regularization to Kantorovich Problem (KP) to obtain a smoothed approximation such as the well-known Sinkhorn algorithm.

We develop this chapter following the intuition that OT can be understood as an optimization problem on different structures. We start from the original OT problem and its duality theorem in Section 2.1. For two Polish probability spaces $\left(\mathcal{X}, P_{X}\right)$ and $\left(\mathcal{Y}, P_{Y}\right)$, Monge Problem (MP) is an optimization problem to find a deterministic map $T: \mathcal{X} \rightarrow \mathcal{Y}$. An equivalent formulation is called $\mathrm{KP}$, which wants to find a joint distribution over all the measures $\mathcal{P}(\mathcal{X} \times \mathcal{Y})$ with fixed marginals. KP is still deterministic. From Riesz representation theorem, we know that there is a duality between a space of bounded signed measures and another space of bounded measurable functions. Similarly, the Duality Problem (DP) of KP is to find a pair of the measurable functions over $L^{1}\left(d P_{X}\right) \times L^{1}\left(d P_{Y}\right)$ on two given measures $d P_{X}$ and $d P_{Y}$. 
Next, we introduce the entropic OT and regularized OT problems, their advantage of unconstrained dual problem and the numerical implementation of the dual problem by Sinkhorn algorithm in Section 2.2. Unlike the problems in previous section, entropic OT and regularized OT are no longer deterministic. The formulations of entropic OT and regularized OT are inherited from the previous $\mathrm{KP}$ and DP, defined on $\mathcal{P}(\mathcal{X} \times \mathcal{Y})$ and $L^{1}\left(d P_{X}\right) \times L^{1}\left(d P_{Y}\right)$.

Then we introduce the time-dependent Monge-Kantorovich problem in Section 2.3. The time-dependent problem points out that the OT map $T$ is differentiable with respect to $t$. Thus, the problem becomes to find a trajectory $\left(T_{t}(x)\right)_{0 \leq t \leq 1}$, where $t \rightarrow T_{t}(x)$ is continuous and piecewise $C^{1}$ for $d \mu$-almost all $x$. The infinitesimal displacement convexity on such trajectory is discussed in Section 2.4 and it leads to HWI inequality. Then, we include Talagrand inequality (Section 2.5) and its application in measure concentration (Section 2.6). In order to introduce the uncertainty of entropic OT, we also go through all the preliminaries needed for our main results, i.e., EPI and deconvolution, in Section 2.8. Furthermore, the time-dependent problem with information constraint can be formulated as a continuous-time stochastic process, which we will discuss in Chapter 4.

\subsection{Optimal Transport}

The OT problem was initialized by Gaspard Monge [23]. The original formulation can be described as follows.

Definition 2.1.1 (MP). [23] Let $P_{X}$ and $P_{Y}$ be two probability measures supported on two Polish space $\mathcal{X}, \mathcal{Y}$. Given a lower semi-continuous (defined in Definition A.5.1) cost function $c(x, y): \mathcal{X} \times \mathcal{Y} \rightarrow \mathbb{R} \cup\{+\infty\}$, we want to find a mapping $T: \mathcal{X} \rightarrow \mathcal{Y}$ minimizing the total cost:

$$
\inf _{T: T_{\#} P_{X}=P_{Y}} \int_{\mathcal{X}} c(x, T(x)) d P_{X}(x) .
$$

Kantorovich showed that there is a more flexible formulation of OT problem. It does not necessarily have to be a deterministic mapping that maps every $x$ to a destination $y$. Instead, every $x$ can correspond to several different destinations of $y$. Hence, Kantorovich gave a probabilistic interpretation to the OT. This is stated next.

Definition 2.1.2 (KP). [24] Let $X$ and $Y$ be two random vectors on two Polish spaces $\mathcal{X}, \mathcal{Y}$. We denote $\mathcal{P}(\mathcal{X})$ and $\mathcal{P}(\mathcal{Y})$ as the sets of all probability 
measures on $\mathcal{X}, \mathcal{Y}$ respectively. Then $X$ and $Y$ have probability measures $P_{X} \in \mathcal{P}(\mathcal{X}), P_{Y} \in \mathcal{P}(\mathcal{Y})$. We denote $\Pi\left(P_{X}, P_{Y}\right)$ as the set of all joint probability measures on $\mathcal{X} \times \mathcal{Y}$ with marginal measures $P_{X}, P_{Y}$. Given a lower semi-continuous cost function $c(x, y): \mathcal{X} \times \mathcal{Y} \rightarrow \mathbb{R} \cup\{+\infty\}$, Kantorovich problem can be written as:

$$
\inf _{P \in \Pi\left(P_{X}, P_{Y}\right)} \int_{\mathcal{X} \times \mathcal{Y}} c(x, y) d P
$$

It can be further proved that (KP) gives the same optimizer as (MP). One can define Wasserstein distance [5, Definition 3.4.1] from KP. Let $\mathcal{X}=\mathcal{Y}$ and let $d$ be a metric on it, Wasserstein distance of order $p, p \geq 1$, is defined as follows,

$$
\mathcal{W}_{p}\left(P_{X}, P_{Y}\right):=\inf _{P \in \Pi\left(P_{X}, P_{Y}\right)}\left[\int_{\mathcal{X} \times \mathcal{Y}} d^{p}(x, y) d P\right]^{\frac{1}{p}} .
$$

Wasserstein distance is a metric between two measures.

Another advantage of (KP) is its duality theorem. Instead of optimizing over all the joint measures, the dual problem requires to optimize over two functions defined on the marginals $d P_{X}$ and $d P_{Y}$ respectively. This is stated next.

Theorem 1 (Kantorovich Duality). [25, Theorem 1.3] Let $\mathcal{X}$ and $\mathcal{Y}$ be two Polish spaces. Let $P_{X} \in \mathcal{P}(\mathcal{X}), P_{Y} \in \mathcal{P}(\mathcal{Y})$ be two given measures. Let $c(x, y): \mathcal{X} \times \mathcal{Y} \rightarrow \mathbb{R}_{+} \cup\{+\infty\}$ be a lower semi-continuous cost function.

For any $P \in \mathcal{P}(\mathcal{X} \times \mathcal{Y})$ and $(\varphi, \psi) \in L^{1}\left(d P_{X}\right) \times L^{1}\left(d P_{Y}\right)$, define

$$
I(P):=\int_{\mathcal{X} \times \mathcal{Y}} c(x, y) d P, \quad J(\varphi, \psi):=\int_{\mathcal{X}} \varphi d P_{X}+\int_{\mathcal{Y}} \psi d P_{Y} .
$$

Define $\Phi_{c}$ to be the set of all measurable functions $(\varphi, \psi) \in L^{1}\left(d P_{X}\right) \times$ $L^{1}\left(d P_{Y}\right)$ satisfying

$$
\varphi(x)+\psi(y) \leq c(x, y),
$$

for $d P_{X}$-almost all $x \in \mathcal{X}$ and $d P_{Y}$-almost all $y \in \mathcal{Y}$. Then

$$
\inf _{\Pi\left(P_{X}, P_{Y}\right)} I(P)=\sup _{\Phi_{c}} J(\varphi, \psi) .
$$

We call the R.H.S of (2.2) the DP. Caffarelli gives an intuitive explanation of DP, which is also called shipper's problem. Imagine that you are a businessman and there are several mines and factories distributed in different locations. You need to transport an amount of coals from the mines to the 
factories. The cost is given by $c(x, y)$ from every starting point $x$ of mines to every destination $y$ of factories for every unit of coals. Another mathematician suggests that he can provide a service that charges $(\varphi(x), \psi(y))$ only at the starting point and the destination respectively and the sum of charges would not exceeds the direct transport cost $c(x, y)$. The DP points out that the total cost of his service can be as much as your initial cost.

Next, we state a famous theorem in OT theory.

Theorem 2 (Brenier's). [25, Theorem 2.12] Let $P_{X} \in \mathcal{P}(\mathcal{X}), P_{Y} \in \mathcal{P}(\mathcal{Y})$ with $\mathcal{X} \subset \mathbb{R}^{n}, \mathcal{Y} \subset \mathbb{R}^{n}$ and assume that $d P_{X}, d P_{Y}$ both have finite second moments. If $P_{X}$ doesn't give mass to small sets, then, for Kantorovich problem with cost $c(x, y)=\frac{1}{2}\|x-y\|^{2}, \exists ! u: \mathcal{X} \rightarrow \mathbb{R}$ gives the optimal coupling

$$
P^{*}=(I d \times \nabla u)_{\#} P_{X},
$$

where $u$ is convex.

We can further show that Brenier's Theorem leads to Monge-Ampère equation. We assume we have density functions $d P_{X}=f(x) d x$ and $d P_{Y}=g(y) d y$. We know that $T$ pushes forward $P_{X}$ to $P_{Y}$, i.e., $\forall y=$ $T(x), f(x) d x=g(y) d y$. Then

$$
\frac{d y}{d x}=\frac{f(x)}{g(y)}
$$

Using the fact that $y=T(x)$, we have

$$
\operatorname{det} D T(x)=\frac{f(x)}{g \circ T(x)} .
$$

From Theorem 2, we know that $T$ is the gradient of a convex function $u$. Therefore, OT problem is equivalent to the following Monge-Ampère equation,

$$
\operatorname{det}\left(\frac{\partial^{2} u}{\partial x_{i} \partial x_{j}}\right)(x)=\frac{f(x)}{g \circ \nabla u(x)} .
$$




\subsection{Entropic OT}

Cuturi in [10] gave the concept of entropic OT. In that definition, he adds an information theoretic constraint to (KP), i.e.,

$$
\inf _{P \in \Pi\left(P_{X}, P_{Y} ; R\right)} \int_{\mathcal{X} \times \mathcal{Y}} c(x, y) d P
$$

where

$$
\Pi\left(P_{X}, P_{Y} ; R\right):=\left\{P \in \Pi\left(P_{X}, P_{Y}\right): I_{P}(X ; Y) \leq R\right\},
$$

with $I_{P}(X ; Y)$ denoting the mutual information [26] between $\mathrm{X}$ and $\mathrm{Y}$.

Similarly, one can define Sinkhorn distance of order $p$ as follows,

$$
\mathcal{W}_{p}\left(P_{X}, P_{Y} ; R\right):=\inf _{P \in \Pi\left(P_{X}, P_{Y} ; R\right)}\left[\int_{\mathcal{X} \times \mathcal{Y}} d^{p}(x, y) d P\right]^{\frac{1}{p}} .
$$

It is obvious that $\Pi\left(P_{X}, P_{Y} ; R\right)$ is a subset of $\Pi\left(P_{X}, P_{Y}\right)$. Because of the minimization problem, it is easy to see that $\mathcal{W}_{p}\left(P_{X}, P_{Y} ; R\right)>\mathcal{W}_{p}\left(P_{X}, P_{Y}\right)$. It is called that entropic OT is a smoothed version of classical OT.

\subsubsection{Existence of solution to $\left(\mathcal{P}_{R}\right)$}

In this subsection, we give a proof of the existence of solution to $\left(\mathcal{P}_{R}\right)$, since $\left(\mathcal{P}_{R}\right)$ is the problem we focus on in our main results.

In order to study the topological and functional property of the constraint set, we first give the definition of weak convergence of probability measures.

Definition 2.2.1 (Weak Convergence of Probability Measures). Let $\mathcal{X}$ be a Polish space. Let $\mathcal{M}(\mathcal{X})$ be a set of all Borel subprobability measures on $\mathcal{X}$, i.e. the set of measures $\gamma$ for which $\gamma(\mathcal{X}) \leq 1$. Let $\mathcal{P}$ be the subset of $\mathcal{M}(\mathcal{X})$ consisting of all probability measures and $C_{b}(\mathcal{X})$ is the set of all bounded continuous functions mapping $\mathcal{X}$ to $\mathbb{R}$. A sequence $\left\{\mu_{n} \in \mathcal{M}(\mathcal{X}), n \in \mathbb{N}\right\}$ weak converges to $\mu$, written as $\mu_{n} \rightarrow \mu$, if for every $g \in C_{b}(\mathcal{X})$,

$$
\lim _{n \rightarrow \infty} \int_{\mathcal{X}} g d \mu_{n}=\int_{\mathcal{X}} g d \mu
$$

A set of measures $\mathcal{P}$ is called sequentially compact if every sequence has a convergent subsequence, i.e., if $\mu_{n} \in \mathcal{P}, n=1,2, \ldots$, then there is a subsequence $n(k)$ of increasing integer and a measure $\mu \in \mathcal{P}$ such that $\mu_{n(k)} \rightarrow \mu$ with respect to weak convergence. Besides directly by its definition, 
an alternative way to prove the compactness is by Prokhorov's theorem. This is stated next.

Theorem 3 (Prokhorov's). [27, Theorem 5.1] Let $\mathcal{X}$ be a Polish space. Let $\mathcal{P}(\mathcal{X})$ be the set of all probability measures on $\mathcal{X}$ and a subset $K \subset \mathcal{P}(\mathcal{X})$. Then $\bar{K}$ is compact if and only if $K$ is tight, i.e. $\forall \epsilon>0$, there exists a compact set $X_{\epsilon} \subset \mathcal{X}$ such that $\mu\left[\mathcal{X} \backslash X_{\epsilon}\right] \leq \epsilon$ for all $\mu \in K$.

Now we can start to prove the existence of $\left(\mathcal{P}_{R}\right)$ with all the preparation we introduced.

Theorem 4 (Existence of solution to $\left(\mathcal{P}_{R}\right)$ ). Let $\mathcal{X}$ and $\mathcal{Y}$ be two Polish spaces, $P_{X} \in \mathcal{P}(\mathcal{X}), P_{Y} \in \mathcal{P}(\mathcal{Y})$. Let $a: \mathcal{X} \rightarrow \mathbb{R} \cup\{-\infty\}$ and $b: \mathcal{X} \rightarrow \mathbb{R} \cup$ $\{-\infty\}$ be two upper semi-continuous functions such that $a \in L^{1}\left(d P_{X}\right)$ and $b \in L^{1}\left(d P_{Y}\right)$. Let $c(x, y): \mathcal{X} \times \mathcal{Y} \rightarrow \mathbb{R}_{+} \cup\{+\infty\}$ be a lower semi-continuous cost function such that $c(x, y) \geq a(x)+b(y)$ for all $x$ and $y$. Then there is a joint distribution $P \in \Pi\left(P_{X}, P_{Y} ; R\right)$ minimizing the total cost $\mathbb{E}_{P}[c(X, Y)]$.

Proof. Because of the lower semi-continuity and further regularity of $c(x, y)$, from [28, Lemma 4.3], we know that the objective function $f: P \rightarrow \int c d P$, is also lower semi-continuous. Hence, according to the extreme value theorem, we only need to show the compactness of $\Pi\left(P_{X}, P_{Y} ; R\right)$ to prove the existence.

We can first prove the tightness of $\Pi\left(P_{X}, P_{Y} ; R\right)$. We know $\mathcal{P}(\mathcal{X}), \mathcal{P}(\mathcal{Y})$ and $\mathcal{B}:=\{P \in \mathcal{P}(\mathcal{X} \times \mathcal{Y}) \mid I(X \| Y) \leq R, 0 \leq R<\infty\}$ are compact [29, 1.4]. Thus for any $\epsilon>0$, by Prokhorov's theorem, there are compact sets $K_{\epsilon} \subset \mathcal{X}, L_{\epsilon} \subset \mathcal{Y}$ and $M_{\epsilon} \subset \mathcal{B}$, such that $\mu\left(\mathcal{X} \backslash K_{\epsilon}\right) \leq \epsilon, \nu\left(\mathcal{Y} \backslash L_{\epsilon}\right) \leq \epsilon$ and $P\left(\mathcal{B} \backslash M_{\epsilon}\right) \leq \epsilon$. Then, for any coupling $(X, Y) \in \Pi\left(P_{X}, P_{Y} ; R\right)$,

$P\left[(X, Y) \notin\left(K_{\epsilon} \times L_{\epsilon}\right) \cap M_{\epsilon}\right] \leq P\left[X \notin K_{\epsilon}\right]+P\left[Y \notin L_{\epsilon}\right]+P\left[(X, Y) \notin M_{\epsilon}\right] \leq 3 \epsilon$.

It is easy to see that $\left(K_{\epsilon} \times L_{\epsilon}\right) \cap M_{\epsilon}$ is compact in $\mathcal{X} \times \mathcal{Y}$. Therefore, $\Pi\left(P_{X}, P_{Y} ; R\right)$ is tight in $\mathcal{P}(\mathcal{X} \times \mathcal{Y})$. By Prokhorov's theorem, this set has a compact closure. $\Pi\left(P_{X}, P_{Y} ; R\right)$ is closed, which means that its closure is itself. So $\Pi\left(P_{X}, P_{Y} ; R\right)$ is compact. Consequently, we finish the proof of the existence to $\left(\mathcal{P}_{R}\right)$.

\subsubsection{Regularized OT}

Entropic OT is a promising way to approximate solutions of Kantorovich problem. However, the hard constraint in $\left(\mathcal{P}_{R}\right)$ is difficult to solve. In order 
to have a simpler, computable formulation, Cuturi introduced a regularized problem in [10], i.e.,

$$
\mathcal{W}_{c, \epsilon}\left(P_{X}, P_{Y}\right):=\inf _{P \in \Pi\left(P_{X}, P_{Y}\right)} \int_{\mathcal{X} \times \mathcal{Y}} c(x, y) d P+\epsilon I(X ; Y),
$$

where $\epsilon \in[0, \infty]$ is a Lagrange multiplier.

Because $\left(\mathcal{P}_{R}\right)$ is a linear programming, the solution is always located on its boundary, i.e., $I(X ; Y)=R$. It can be immediately shown that $\left(\mathcal{P}_{R}\right)$ and $\left(\mathcal{P}_{\epsilon}\right)$ give the same optimizer $P$ by the duality theorem. In our main results, we study $\left(\mathcal{P}_{R}\right)$ instead of $\left(\mathcal{P}_{\epsilon}\right)$ for two reasons. First, the rate $R$ in $\left(\mathcal{P}_{R}\right)$ describes the amount of uncertainty. Second, the expectation of cost function has a more explicit physical meaning.

Unlike (MP) and (KP), there is no more deterministic map anymore for $\left(\mathcal{P}_{R}\right)$ and $\left(\mathcal{P}_{\epsilon}\right)$, because an one-to-one mapping leads to an infinite mutual information. From Figure 2.1a to 2.1c, we plot the color map of the optimizer $P^{*}$ for $\left(\mathcal{P}_{R}\right)$ between two Gaussian marginals with respect to different values of parameter $R$, which is equivalent to $\left(\mathcal{P}_{\epsilon}\right)$ with some specific values of $\epsilon$. We can observe that the uncertainty decreases, i.e., the joint probability of two random variables concentrates into a smaller area, when we increase the value of $R$. When $R \rightarrow+\infty,\left(\mathcal{P}_{R}\right)$ is unconstrained with mutual information and becomes classic (KP). When $R=0$, the two random variables are independent.

From the computational perspective, the well-known Sinkhorn algorithm computes $\left(\mathcal{P}_{\epsilon}\right)$. By dual formulation, it leverages the computation to logarithmic to obtain an approximation of optimal transport distance. The experimental results of Sinkhorn show that the smooth approximation works better than other metrics when computing the distance of noisy observations on manifold [10].

Here we introduce a more general regularization by $f$-divergence. As a special case, the duality of entropic OT will immediately follow after the general duality of regularized OT.

Definition 2.2.2 ( $f$-divergence). Let $\mathcal{X}$ be a Polish space. Let $f$ be a convex, lower semi-continuous function such that $f(1)=0$. The $f$ divergence $D_{f}(\cdot \| \cdot)$ of two probability measures $\mu, \nu \in \mathcal{P}(\mathcal{X})$ is defined by:

$$
D_{f}(\mu \| \nu):=\int_{\mathcal{X}} f\left(\frac{d \mu}{d \nu}\right) d \nu+f_{\infty} \mu^{\perp}(\mathcal{X})
$$

where $f_{\infty}:=\lim _{x \rightarrow+\infty} f(x) / x$ and $\mu^{\perp}(\mathcal{X})$ is the part of measure that is not 


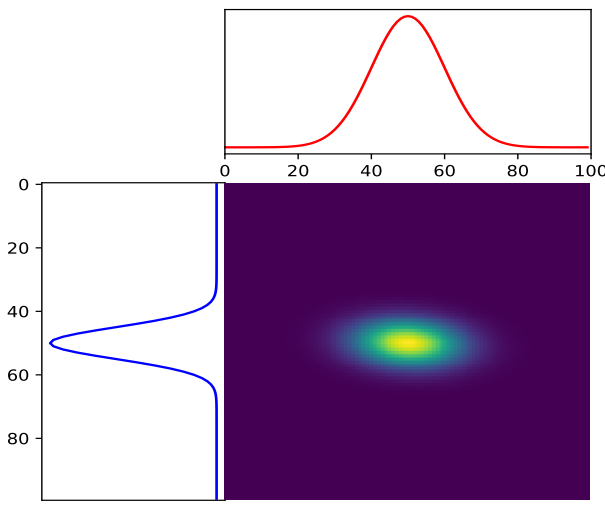

(a) $R=0.0051$ or $\epsilon=0.1$.

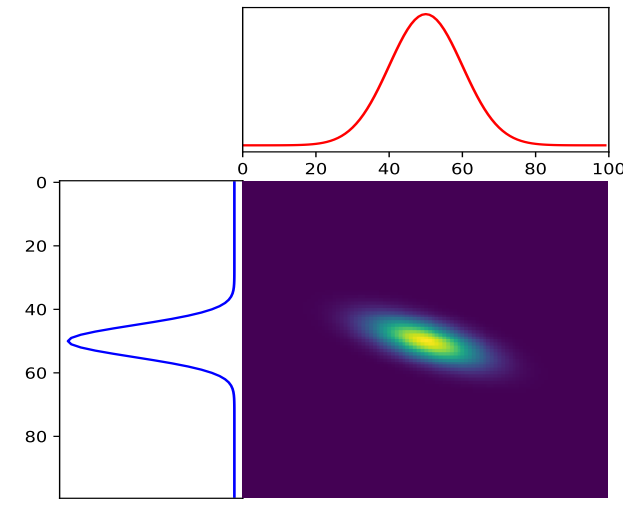

(b) $R=0.25$ or $\epsilon=0.01$.

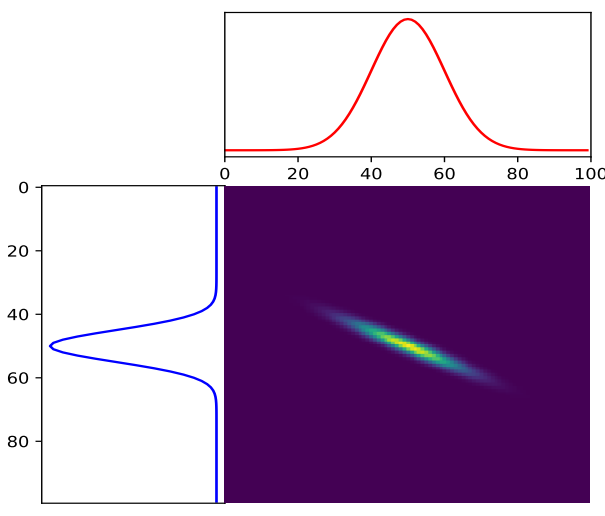

(c) $R=1.19$ or $\epsilon=0.001$.

Figure 2.1 - Color maps of the optimizer $P^{*}$ with respect to different $R$ for $\left(\mathcal{P}_{R}\right)$, or equivalently, different $\epsilon$ for $\left(\mathcal{P}_{\epsilon}\right)$. 
absolutely continuous with respect to $d \mu$ in the Radon-Nikodym decomposition, i.e., $d \mu=\frac{d \mu}{d \nu}(x) d \nu+d \mu^{\perp}$.

$f$-divergences characterize the difference between two probability measures. The KL-divergence takes $f(x)=x \log x$. However, $f$-divergence doesn't metrize weak-convergence. For example, assume a sequence of Dirac functions $\mu_{n}=\delta(x-1 / n)$ and $\mu=\delta(x)$. We have $\mu_{n} \rightarrow \mu$. But $D\left(\mu_{n} \| \mu\right)=+\infty$ for any $n$, which doesn't imply the weak-convergence of $\mu_{n}$.

Then we give the definition of regularized OT penalized by $f$-divergence.

Definition 2.2.3 (Regularized OT). Let $\mathcal{X}$ and $\mathcal{Y}$ be two Polish spaces, $P_{X} \in$ $\mathcal{P}(\mathcal{X}), P_{Y} \in \mathcal{P}(\mathcal{Y})$. Let $c(x, y): \mathcal{X} \times \mathcal{Y} \rightarrow \mathbb{R}_{+} \cup\{+\infty\}$ be a lower semicontinuous cost function and $f: \mathbb{R}_{+} \rightarrow \mathbb{R}$ be a convex, lower semi-continuous function. Define regularized optimal transport $\left(\mathcal{P}_{\epsilon, f}\right)$ as

$$
\begin{aligned}
\mathcal{W}_{c, \epsilon}^{f}\left(P_{X}, P_{Y}\right):=\inf _{P \in \Pi\left(P_{X}, P_{Y}\right)} & \int_{\mathcal{X} \times \mathcal{Y}} c(x, y) d P \\
& +\epsilon \int_{\mathcal{X} \times \mathcal{Y}} f\left(\frac{d P}{d P_{X} \otimes P_{Y}}\right) d P_{X} \otimes P_{Y}, \quad\left(\mathcal{P}_{\epsilon, f}\right)
\end{aligned}
$$

where $P_{X} \otimes P_{Y} \in \mathcal{P}(\mathcal{X} \times \mathcal{Y})$ is a product measure.

Similar to Theorem 1, we can also give a dual formulation of regularized OT, which is subject to two functionals defined to the marginal measures.

Theorem 5 (Strong Duality of Regularized OT). [30] The dual problem of the primal regularized OT $\left(\mathcal{P}_{\epsilon, f}\right)$ is as follows,

$$
\begin{aligned}
\mathcal{W}_{c, \epsilon}^{f}\left(P_{X}, P_{Y}\right) & =\sup _{(\varphi, \psi) \in L^{1}(\mathcal{X}) \times L^{1}(\mathcal{Y})} \int_{\mathcal{X}} \varphi d P_{X}+\int_{\mathcal{Y}} \psi d P_{Y} \\
& -\epsilon \int_{\mathcal{X} \times \mathcal{Y}} f^{*}\left(\frac{\varphi(x)+\psi(y)-c(x, y)}{\epsilon}\right) d P_{X} \otimes P_{Y}, \quad\left(\mathcal{D}_{\epsilon, f}\right)
\end{aligned}
$$

where $f^{*}$ is the Legendre transform of $f$ defined by $f^{*}(y):=\sup _{x} x y-f(x)$. The primal-dual relationship is given by

$$
P=\underset{P \in \Pi\left(P_{X}, P_{Y}\right)}{\arg \min } f\left(\frac{d P}{d P_{X} \otimes P_{Y}}\right)-\frac{\varphi(x)+\psi(y)-c(x, y)}{\epsilon} \frac{d P}{d P_{X} \otimes P_{Y}},
$$

where $P$ is the solution of $\left(\mathcal{P}_{\epsilon, f}\right)$ and $(\varphi, \psi)$ is the solution of $\left(\mathcal{D}_{\epsilon, f}\right)$. The relationship can be written equivalently as

$$
d P=\left(f^{\prime}\right)^{-1}\left(\frac{\varphi(x)+\psi(y)-c(x, y)}{\epsilon}\right) d P_{X} \otimes P_{Y},
$$


when the derivative $f^{\prime}$ is invertible.

Remark. The existence of solution of $\left(\mathcal{P}_{\epsilon, f}\right)$ is proved in [31, Prop. 3.5.6]. $\left(\mathcal{D}_{\epsilon, f}\right)$ doesn't always guarantee a maximizer. However, the proof of existence of entropic optimal transport, where $f$ is restricted as $x \log x$, is given in [30, Theorem 7].

Let $f=x \log x-x+1$. Because the two measures have the same total volume, the $f$-divergence is still KL-divergence. Thus, we obtain the dual formulation of $\left(\mathcal{P}_{\epsilon}\right)$.

Corollary 5.1 (Duality of Entropic Optimal Transport). $\left(\mathcal{P}_{\epsilon}\right)$ is equivalent to the following problem,

$$
\begin{aligned}
& \mathcal{W}_{c, \epsilon}\left(P_{X}, P_{Y}\right) \\
= & \sup _{(\varphi, \psi) \in L^{1}(\mathcal{X}) \times L^{1}(\mathcal{Y})} \int_{\mathcal{X}} \varphi d P_{X}+\int_{\mathcal{Y}} \psi d P_{Y}-\epsilon \int_{\mathcal{X} \times \mathcal{Y}} e^{\frac{\varphi(x)+\psi(y)-c(x, y)}{\epsilon}} d P_{X} \otimes d P_{Y}+\epsilon \\
= & \sup _{(\varphi, \psi) \in L^{1}(\mathcal{X}) \times L^{1}(\mathcal{Y})} \mathbb{E}_{P}\left[f_{\epsilon}^{X Y}(\varphi, \psi)\right]+\epsilon,
\end{aligned}
$$

where $f_{\epsilon}^{X Y}(\varphi, \psi)=\varphi(x)+\psi(y)-\epsilon e^{\frac{\varphi(x)+\psi(y)-c(x, y)}{\epsilon}}$. The primal-dual relationship is given by

$$
d P=e^{\frac{\varphi(x)+\psi(y)-c(x, y)}{\epsilon}} d P_{X} \otimes P_{Y} .
$$

\subsubsection{Sinkhorn Algorithm}

We can see that there is no constraint of marginals in the dual problem $\left(\mathcal{D}_{\epsilon, f}\right)$, compared to DP in standard OT. Both variables are concave in $\left(\mathcal{D}_{\epsilon}\right)$, thus we can fix one and optimize over the other. Using the first order condition of $\varphi$ in $\left(\mathcal{D}_{\epsilon}\right)$, we have

$$
\varphi(x)=-\epsilon \log \left(\int_{\mathcal{Y}} e^{\frac{\psi(y)-c(x, y)}{\epsilon}} d P_{Y}\right)
$$

Similarly, for $\psi$ :

$$
\psi(y)=-\epsilon \log \left(\int_{\mathcal{X}} e^{\frac{\varphi(x)-c(x, y)}{\epsilon}} d P_{X}\right)
$$

$(\varphi, \psi)$ solve a fixed point equation. We rewrite the optimality condition. Let $(a, b)$ be the exponential scalings of $(\varphi, \psi)$, i.e., $a(x)=e^{\varphi(x) / \epsilon}$ and $b(y)=$ 
$e^{\psi(y) / \epsilon}$. Let $\mathcal{L}$ and $\mathcal{L}^{\prime}$ be two operators such that

$$
\mathcal{L}(f):=\left(\int_{\mathcal{X}} f(x) e^{\frac{-c(x, y)}{\epsilon}} d P_{X}\right)^{-1} \quad \text { and } \quad \mathcal{L}^{\prime}(f):=\left(\int_{\mathcal{Y}} f(y) e^{\frac{-c(x, y)}{\epsilon}} d P_{Y}\right)^{-1} .
$$

Then the fixed point equation can be further simplified: the optimal exponential scalings $\left(a^{*}, b^{*}\right)$ satisfy the follows,

$$
a^{*}=\mathcal{L}^{\prime} \circ \mathcal{L}\left(a^{*}\right) \quad \text { and } \quad b^{*}=\mathcal{L} \circ \mathcal{L}^{\prime}\left(b^{*}\right)
$$

Let $P_{X}$ and $P_{Y}$ be discrete measures on finite supports, i.e., $d P_{X}=\sum_{i=1}^{n} \boldsymbol{\alpha}_{i} \delta_{x_{i}}$ and $d P_{Y}=\sum_{i=1}^{n} \boldsymbol{\beta}_{i} \delta_{y_{i}}$, where $\left\{x_{i}\right\}$ and $\left\{y_{i}\right\}$ are two sets of points on $\mathbb{R}^{n}$. Then the fixed point iteration exactly corresponds to the well-known Sinkhorn algorithm [32, 33, 34]. Let $\boldsymbol{a}_{i}=a\left(x_{i}\right), \boldsymbol{b}_{i}=b\left(x_{i}\right)$ and $\boldsymbol{K}_{i, j}=e^{\frac{-c\left(x_{i}, y_{j}\right)}{\epsilon}}$. The Sinkhorn iteration is given by

$$
\boldsymbol{a}^{(l+1)}=\frac{1}{\boldsymbol{K}\left(\boldsymbol{b}^{(l)} \odot \boldsymbol{\beta}\right)} \quad \text { and } \quad \boldsymbol{b}^{(l+1)}=\frac{1}{\boldsymbol{K}^{\top}\left(\boldsymbol{a}^{(l)} \odot \boldsymbol{\alpha}\right)},
$$

where $\boldsymbol{a} \odot \boldsymbol{b}$ is Hadamard product and where the division is element-wise. $\left(\boldsymbol{a}^{(l)}, \boldsymbol{b}^{(l)}\right)$ converge to the exponential scalings of a solution $\left(\boldsymbol{a}^{*}, \boldsymbol{b}^{*}\right)$ of the dual problem $\left(\mathcal{D}_{\epsilon, f}\right)$. The optimal transport plan is given by

$$
\boldsymbol{P}^{*}=\operatorname{diag}\left(\boldsymbol{a}^{*} \odot \boldsymbol{\alpha}\right) \boldsymbol{K} \operatorname{diag}\left(\boldsymbol{b}^{*} \odot \boldsymbol{\beta}\right) .
$$

We can also write the iteration as the following pseudocode.

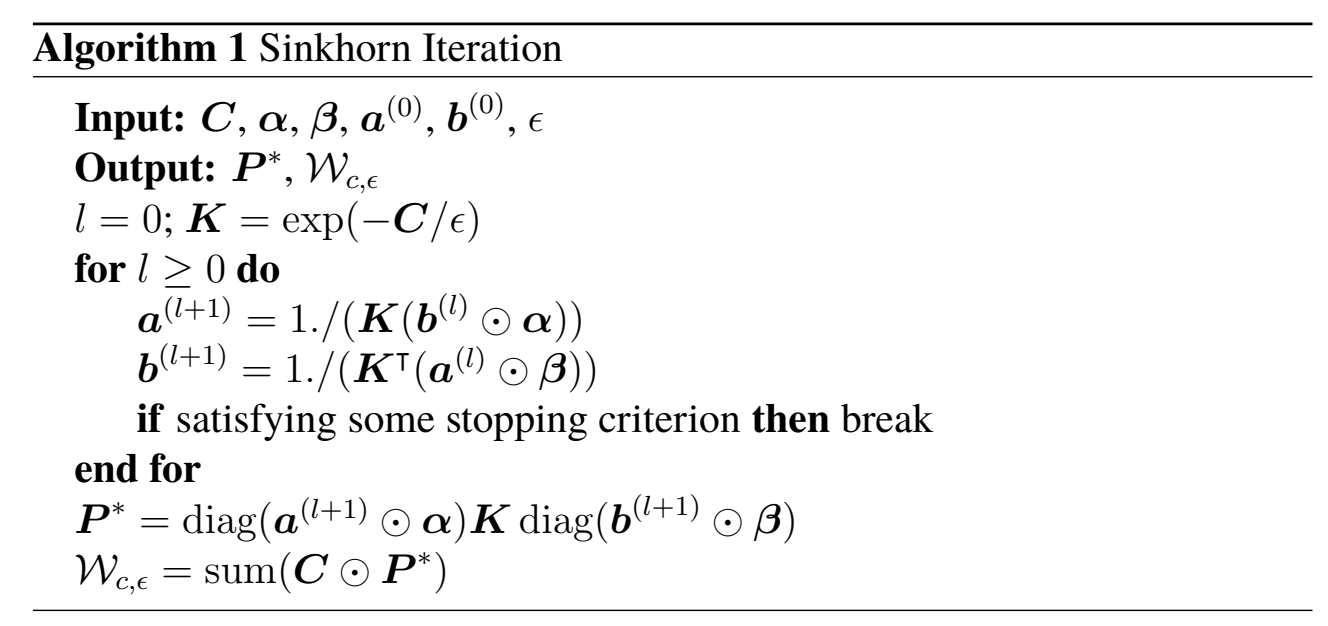

The complexity of each iteration is $\mathcal{O}\left(n^{2}\right)$ if $(\boldsymbol{a}, \boldsymbol{b})$ have the same length $n$. It is a major improvement compared to the complexity of $\mathcal{O}\left(n^{3} \log n\right)$ to 
solve the linear programming for standard discrete OT. The convergence rate of Sinkhorn iterations can be found in [35].

There is another kind of approximation method for linear programming called interior point method [36], which provides a faster convergence rate. It uses a barrier function as penalty, which tends to infinity along a sequence of interior point converging to its boundary points. We can see $I(X ; Y)$ is not such a barrier function, since it is not infinite on its boundary.

\subsection{Time-dependent Monge-Kantorovich Problem}

Many physics and engineering problems are time-dependent, for example, to study how the dynamics of a system develop during an interval of time. If generalizing the Monge-Kantorovich problem into a time-dependent problem, we can see that the original distance problem (find the distance between two probability measures $\mu$ and $\nu$ ) changes into a geodesic problem (find the optimal path between $\mu$ and $\nu$ ).

In order to model this time-dependent problem, we introduce the trajectory $\left(T_{t}(x)\right)_{0 \leq t \leq 1}$, which we shall often abbreviate as $\left(T_{t}(x)\right) . C\left[T_{t}(x)\right]$ becomes the displacement cost for transport from $x$ to $y$. We assume $t \rightarrow T_{t}(x)$ to be continuous and piecewise $C^{1}$ for $d \mu$-almost all $x$. Then we have timedependent minimization problem

$$
\inf \left\{\int_{\mathcal{X}} C\left[\left(T_{t}(x)\right)_{0 \leq t \leq 1}\right] d \mu(x): T_{0}=I d, T_{1 \#} \mu=\nu\right\} \text {. }
$$

(2.6) is compatible with (MP). A simple sufficient condition for compatibility is

$$
c(x, y)=\inf \left\{C(z(t))_{0 \leq t \leq 1}: z(0)=x, z(1)=y\right\},
$$

where $z(t)$ is the trajectory with fixed starting point $x$ and destination $y$.

Theorem 6 (Time-dependent OT Theorem). [25, Theorem 5.5] Consider a given cost function $c(x, y)=c(x-y)$ on $\mathbb{R}^{n}$, with $c$ strictly convex, $c(0)=0$. Let $\mu, \nu \in \mathcal{P}\left(\mathbb{R}^{n}\right)$ be two probability measures absolutely continuous with respect to Lebesgue measure. Let $C\left[\left(z_{t}\right)\right]=\int_{0}^{1} c\left(\dot{z}_{t}\right) d t$. Let $\nabla \varphi$ be the ( $d \mu$-almost everywhere) unique gradient of a $c$-concave (see Definition A.5.7) function $\varphi$ such that $\left[I d-\nabla c^{*}(\nabla \varphi)\right]_{\#} \mu=\nu$. Then the solution of (2.6) is given by

$$
T_{t}(x)=x-t \cdot \nabla c^{*}(\nabla \varphi(x)), \quad 0 \leq t \leq 1,
$$

where $c^{*}$ is the Legendre transformation of $c$. 
Proof. It is easy to see that the interpolation between $T_{0}(x)$ and $T_{1}(x)$ should be linear on $\mathbb{R}^{n}$, i.e., $T_{t}(x)=(1-t) T_{0}(x)+t T_{1}(x)$. This leads to equation (2.7).

Remark. The assumption $\left[I d-\nabla c^{*}(\nabla \varphi)\right]_{\#} \mu=\nu$ is from the twist condition $\nabla \varphi(x)-\nabla_{x} c(x-y)=0$ (cf. [28, Theorem 10.28]). From the Euler's derivative notation of Legendre transformation $\nabla f(\cdot)=\left(\nabla f^{*}\right)^{-1}(\cdot)$, the equivalence between the assumption and twist condition is immediately shown.

We look into the quadratic cost case. Let $c(x-y)=\frac{1}{2}\|x-y\|^{2}$, then the total cost can be represented by the differentiable cost

$$
\frac{1}{2}\|x-y\|^{2}=\inf _{z(t) \in \mathcal{Z}_{x y}} \int_{0}^{1} \frac{1}{2}\|\dot{z}(t)\|^{2} d t,
$$

where $\mathcal{Z}_{x y}$ is the family of $C^{1}\left([0,1] ; \mathbb{R}^{n}\right)$ trajectories with $z(0)=x$ and $z(1)=$ $y$. For quadratic cost, $\nabla c^{*}(\nabla \varphi(x))=\nabla \varphi(x)$. Therefore, according to (2.7), the optimal trajectory $z^{*}(t)$ satisfies

$$
\dot{z}^{*}(t)=-\nabla \varphi(x) .
$$

The OT problem also admits a dynamical formulation, aka BenamouBrenier Problem [37]. Let $\rho(x, t)$ be the density field of a mass of particles $z$ 's such that $d \mu(z)=\rho(z, 0), d \nu(z)=\rho(z, 1)$ and $z^{v}(t) \sim \rho(z, t)$. Let $v\left(t, z^{v}(t)\right)$ be the velocity field such that

$$
\dot{z}^{v}(t)=v\left(t, z^{v}(t)\right)
$$

Let $c(x-y)=\frac{1}{2}\|x-y\|^{2}$, then (2.6) can be written as

$$
\inf _{v \in \mathcal{V}}\left\{\int_{[0,1] \times \mathbb{R}^{n}} \frac{1}{2}\left\|v\left(t, z^{v}(t)\right)\right\|^{2} \rho(z, t) d z d t\right\}
$$

where $\mathcal{V}$ is the set of $v$ satisfying

$$
\begin{aligned}
& \dot{\rho}+\nabla \cdot(v \rho)=0, \\
& \rho(\cdot, 0)=\rho_{0}, \rho(\cdot, 1)=\rho_{1} .
\end{aligned}
$$

(2.9) is the continuity equation. Benamou-Brenier Problem is defined by (2.8), (2.9) and (2.10).

In Figure 2.2a and 2.2b, we plot the distributions uniformly sampled from geodesics, i.e. $\rho\left(\cdot, \frac{k}{n}\right), k=0,1, \ldots, n, n \in \mathbb{N}$, with respect to 2 -Wasserstein 


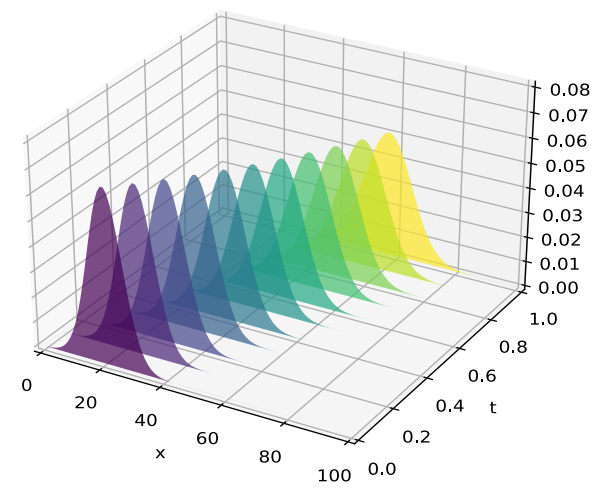

(a) Wasserstein distance.

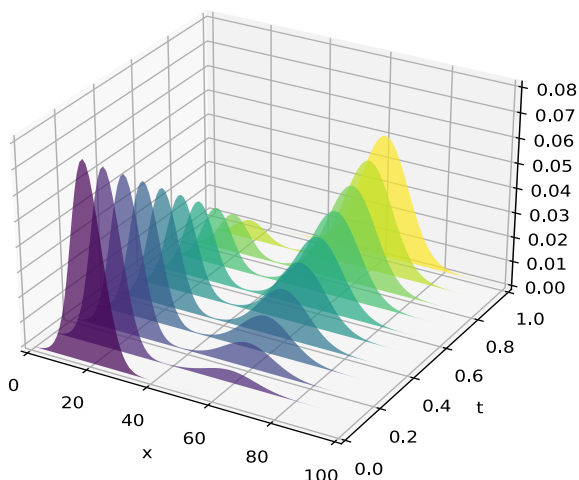

(b) L2 norm.

Figure 2.2 - Interpolations between two Gaussian distributions by different metrics.

distance and L2 norm. We know that the Wasserstein geodesic is point to point linear, according to (2.7). Thus, the Wasserstein interpolation between two Gaussian in Figure 2.2a looks more reasonable and preserves the distributions Gaussian.

\subsection{Infinitesimal Displacement Convexity}

In this section, we start with a lower bound for a derivative of $U_{\nu}\left(\mu_{t}\right)$, where $U_{\nu}$ is an energy functional with a reference measure $\nu$ and $\left(\mu_{t}\right)_{0 \leq t \leq 1}$ is a Wasserstein geodesic. We will show that it leads to HWI inequality, which is an important functional inequality and unifies the Talagrand inequality and logarithmic Sobolev inequality.

Consider a locally compact, complete geodesic space $\mathcal{X}$ equipped with a local finite measure $\mu$ and a distance $d$. Then for a convex function $U$ : $[0,+\infty) \rightarrow \mathbb{R}$, twice differentiable on $(0,+\infty)$, the functional of energy associated to $U$ is defined by

$$
U_{\nu}(\mu):=\int_{\mathcal{X}} U(\rho) d \nu, \quad \mu=\rho \nu .
$$

Theorem 7 (Differentiating an energy along OT). [28, Theorem 20.1] Let $(\mathcal{X}, d, \nu)$ be a Polish space with a reference measure $\nu$ and let $U$ be as above. Let $\left(\mu_{t}\right)_{0 \leq t \leq 1}$ be a geodesic in $\mathcal{P}(\mathcal{X})$ such that each $\mu_{t}$ is absolutely continuous with respect to $\nu$ with density $\rho_{t}$ and $U\left(\rho_{t}\right)_{-}$is $\nu$-integrable for all $t$. Further 
assume that $\rho_{0}$ is Lipschitz continuous, $U\left(\rho_{0}\right)$ and $\rho_{0} U^{\prime}(\rho)$ is $\nu$-integrable, and $U^{\prime}$ is Lipschitz continuous on $\rho_{0}(\mathcal{X})$. Then

$$
\liminf _{t \rightarrow 0} \frac{U_{\nu}\left(\mu_{t}\right)-U_{\nu}\left(\mu_{0}\right)}{t} \geq-\int_{\mathcal{X}} U^{\prime \prime}\left(\rho_{0}\left(x_{0}\right)\right)\left\|\nabla^{-} \rho_{0}\right\|\left(x_{0}\right) d\left(x_{0}, x_{1}\right) \pi\left(d x_{0} d x_{1}\right),
$$

where $\left\|\nabla^{-} f\right\|(x):=\limsup _{y \rightarrow x} \frac{[f(y)-f(x)]_{-}}{d(x, y)}$ and $a_{-}:=\max (-a, 0) . \pi$ is an optimal coupling of $\left(\mu_{0}, \mu_{1}\right)$ associated with the geodesic $\left(\mu_{t}\right)_{0 \leq t \leq 1}$.

Consider a Riemannian manifold $M$, equipped with a reference measure $\nu$ and satisfying $\operatorname{CD}(0, \infty)$. From [28, Theorem 17.15], we know the energy functional $U_{\nu}$ is displacement convex on $M$. That is, if $\left(\mu_{t}\right)_{0 \leq t \leq 1}$ is a geodesic in $\mathcal{P}^{a c}(\mathcal{X})$, for $0 \leq t \leq 1$ we have the convexity inequality

$$
U_{\nu}\left(\mu_{t}\right) \leq(1-t) U_{\nu}\left(\mu_{0}\right)+t U_{\nu}\left(\mu_{1}\right)
$$

which may be rewritten as

$$
\frac{U_{\nu}\left(\mu_{t}\right)-U_{\nu}\left(\mu_{0}\right)}{t} \leq U_{\nu}\left(\mu_{1}\right)-U_{\nu}\left(\mu_{0}\right)
$$

Under certain regularities, we let $U(\rho)=\rho \log \rho$ and apply Theorem 7 to pass to the limit as $t \rightarrow 0$. Then

$$
-\int_{\mathcal{X}} \frac{\left\|\nabla \rho_{0}\left(x_{0}\right)\right\|}{\rho_{0}\left(x_{0}\right)} d\left(x_{0}, x_{1}\right) \pi\left(d x_{0} d x_{1}\right) \leq U_{\nu}\left(\mu_{1}\right)-U_{\nu}\left(\mu_{0}\right) .
$$

Using Cauchy-Schwarz inequality, this further implies

$$
\begin{aligned}
U_{\nu}\left(\mu_{0}\right)-U_{\nu}\left(\mu_{1}\right) & \leq \sqrt{\int \frac{\left\|\nabla \rho_{0}\left(x_{0}\right)\right\|^{2}}{\rho_{0}\left(x_{0}\right)^{2}} \pi\left(d x_{0} d x_{1}\right)} \sqrt{d\left(x_{0}, x_{1}\right)^{2} \pi\left(d x_{0} d x_{1}\right)} \\
& =\mathcal{W}_{2}\left(\mu_{0}, \mu_{1}\right) \sqrt{\frac{\left\|\nabla \rho_{0}\right\|^{2}}{\rho_{0}} d \nu}
\end{aligned}
$$

In this Riemannian manifold case, (2.12) is the HWI inequality, which gives a relation between KL-divergence $D(\mu \| \nu)=U_{\nu}(\mu)$ (also written as $H(\mu \mid \nu)$ in physics), Wasserstein distance $\mathcal{W}$ and relative Fisher information $I\left(\mu_{0} \mid \nu\right):=$ $\sqrt{\frac{\left\|\nabla \rho_{0}\right\|^{2}}{\rho_{0}} d \nu}$.

Furthermore, we know that the Ricci curvature of a Euclidean space equipped with a strongly log-concave reference measure is always positive. Therefore, there are stronger HWI inequalities on such spaces. 
Theorem 8 (HWI Inequality). [38] Let $d \nu=e^{-V}$ be a reference probability distribution on $\mathbb{R}^{n}$ such that $D^{2} V \geq \lambda I_{n}$ for some $\lambda>0$. Then $\forall \mu \in \mathcal{P}^{a c}(\mathcal{X})$,

$$
D(\mu \| \nu) \leq \mathcal{W}_{2}(\mu, \nu) \sqrt{I(\mu \mid \nu)}-\frac{\lambda}{2} \mathcal{W}_{2}^{2}(\mu, \nu)
$$

A more general inequality later appears in the work of Otto [39]. It states that for all $\mu_{0}, \mu_{1} \in \mathcal{P}^{a c}(\mathcal{X})$,

$$
D\left(\mu_{0} \| \nu\right) \leq D\left(\mu_{1} \| \nu\right)+\mathcal{W}_{2}\left(\mu_{0}, \mu_{1}\right) \sqrt{I\left(\mu_{0} \mid \nu\right)}-\frac{\lambda}{2} \mathcal{W}_{2}^{2}\left(\mu_{0}, \mu_{1}\right) .
$$

(2.14) recovers (2.13) immediately if we let $\mu_{1}=\nu$. Moreover, to recover Talagrand inequality (2.15), we let $\mu=\nu$ in (2.13).

\subsection{Talagrand Inequality}

Following the results of HWI inequality, we state some known results of Talagrand inequality [1] in this section.

Definition 2.5.1 (Talagrand Inequality). Let $P_{X}$ be a reference probability measure with density $e^{-V(x)}$. We say $P_{X}$ satisfies $\boldsymbol{T}(\lambda)>0$, i.e., Talagrand inequality with parameter $\lambda>0$, if for any $P_{Y} \in \mathcal{P}(\mathcal{Y})$,

$$
\mathcal{W}_{2}\left(P_{X}, P_{Y}\right) \leq \sqrt{\frac{2}{\lambda} D\left(P_{Y} \| P_{X}\right)}
$$

Remark. (2.15) shows that KL-divergence has a stronger convergence than quadratic Wasserstein distance. It was originally introduced by Talagrand [1] when $P_{X}$ is Gaussian. Blower [40] gave a refinement and proved that

$$
D^{2} V \geq \lambda I_{n} \quad \Rightarrow \quad \boldsymbol{T}(\lambda)
$$

There is a stronger statement in the slightly earlier work [38]:

$$
\operatorname{LSI}(\lambda) \Rightarrow \boldsymbol{T}(\lambda),
$$

where $\operatorname{LSI}(\lambda)$ is the logarithmic Sobolev inequality with parameter $\lambda$ (see Definition 2.6.1).

Recently, new inequalities with dimensional improvements were obtained. These dimensional improvements were first observed in the Gaussian case of logarithmic Sobolev inequality, Brascamp-Lieb (or Poincaré) inequality 
[41] and Talagrand inequality [2]. For a standard Gaussian measure $P_{X}$, the dimensional Talagrand inequality has the form:

$$
\mathcal{W}_{2}^{2}\left(P_{X}, P_{Y}\right) \leq \mathbb{E}\left[\|Y\|^{2}\right]+n-2 n e^{\frac{1}{2 n}\left(\mathbb{E}\left[\|Y\|^{2}\right]-n-2 D\left(P_{Y} \| P_{X}\right)\right)} .
$$

Bolley et al. in [4] generalized the results in [41, 2] from Gaussian to strongly log-concave or log-concave. Next, we state his result. Let $d P_{X}=e^{-V}$, where $V: \mathbb{R}^{n} \rightarrow \mathbb{R}$ is $C^{2}$ continuous, $D^{2} V \geq \lambda I_{n}$. Bolley's dimensional Talagrand inequality is given as follows,

$$
\frac{\lambda}{2} \mathcal{W}_{2}^{2}\left(P_{X}, P_{Y}\right) \leq \mathbb{E}[V(Y)]-\mathbb{E}[V(X)]+n-n e^{\frac{1}{n}\left(\mathbb{E}[V(Y)]-\mathbb{E}[V(X)]-D\left(P_{Y} \| P_{X}\right)\right)}
$$

Bai et al. in [11] gave a generalization of (2.16) to Sinkhorn distance. When $P_{X}$ is standard Gaussian,

$$
\mathcal{W}_{2}^{2}\left(P_{X}, P_{Y} ; R\right) \leq \mathbb{E}\left[\|Y\|^{2}\right]+n-2 n \sqrt{\frac{1}{2 \pi e}\left(1-e^{-\frac{2}{n} R}\right)} e^{\frac{1}{n} h(Y)} .
$$

When $R \rightarrow+\infty$, this inequality coincides with (2.16), which is tighter than (2.15).

\subsection{Measure Concentration}

Measure concentration phenomenon describes how the probability of a random variable $X$ changes with the deviation from a given value like its mean or median. Concentration-of-measure inequalities give bounds for measure concentration phenomenon. They are powerful tools used in various areas, including convex geometry, physics, statistics, theoretical computer science, information theory and learning theory.

Most concentration-of-measure inequalities bound the probability of a given function $f\left(X^{n}\right)$ of a randon $n$-tuple $X^{n} \in \mathcal{X}^{n}$. We next introduce one such inequality obtained by Gaussian log-Sobolev inequality.

We first give the formal statement for log-Sobolev inequality.

Definition 2.6.1 (Log-Sobolev inequality). [42, Definition 5.1.1] Let $P_{X} \in$ $\mathcal{P}(\mathcal{X})$ be a reference probability measure. We say $P_{X}$ satisfies $\operatorname{LSI}(\lambda)$, i.e., $\log$-Sobolev inequality with parameter $\lambda>0$, if for any smooth $f: \mathcal{X} \rightarrow \mathbb{R} \in$ 
$L^{2}(\mathcal{X})$

$$
\mathbb{E}\left[f^{2}(X) \ln f^{2}(X)\right]-\mathbb{E}\left[f^{2}(X)\right] \ln \mathbb{E}\left[f^{2}(X)\right] \leq 2 \lambda \mathbb{E}\left[\|\nabla f(X)\|^{2}\right] .
$$

WLOG, we can fix the function $f$ satisfying $\int_{\mathcal{X}} f^{2} d P_{X}=1$ and let $d P_{Y}=$ $f^{2} d P_{X}$. Then we get an alternative formulation for log-Sobolev inequality. For every $P_{Y} \in \mathcal{P}(\mathcal{X}) \ll P_{X}$,

$$
D\left(P_{Y} \| P_{X}\right) \leq \frac{\lambda}{2} I\left(P_{Y} \mid P_{X}\right) .
$$

Gaussian log-Sobolev inequality is the log-Sobolev inequality with $P_{X}$ as Gaussian and $\lambda=1$. It leads to the following proposition of measure concentration.

Proposition 9. [5, Proposition 3.2.2] Let $X^{n} \sim \mathcal{N}\left(0, I_{n}\right)$. Then we have, for every smooth function $f: \mathbb{R}^{n} \rightarrow \mathbb{R}$ such that $\left\|\nabla f\left(X^{n}\right)\right\| \leq 1$ almost surely,

$$
P\left(f\left(X^{n}\right) \geq \mathbb{E}\left[f\left(X^{n}\right)\right]+r\right) \leq e^{-\frac{r^{2}}{2}}, \quad \forall r \geq 0 .
$$

Unlikes the concentration of a functional defined on a measure in (2.20), Marton introduced an approach of concentration directly on the level of probability measures (see $[5,3.4 .2]$ ). The intuition behind is that optimal transport theory can give a metric of two probability measures by the based metric structure of a Polish space. The metric can be further connected with probability divergence by Talagrand inequality.

To give the notation of concentration of measure, we first introduce metric probability space. Let $(\mathcal{X}, d)$ be a Polish space and $\mu$ is a probability measure defined on the Borel set of $(\mathcal{X}, d)$. Then we say the triple $(\mathcal{X}, d, \mu)$ is a metric probability space.

For an arbitrary set $A \subset \mathcal{X}$ and any $r \geq 0$, we define $A_{r}$ as

$$
A_{r}:=\{x \in \mathcal{X}: d(x, A)>r\},
$$

where $d(x, A):=\inf _{a \in A} d(x, a)$. Then we say a probability measure has normal (or Gaussian) concentration on $(\mathcal{X}, d)$ if there exist positive $K$ and $\kappa$ such that

$$
\mu(A) \geq \frac{1}{2} \Rightarrow \mu\left(A_{r}\right) \leq K e^{-\kappa r^{2}}, \forall r>0
$$


There is another weaker statement of normal concentration, such that

$$
\mu(A) \geq \frac{1}{2} \Rightarrow \mu\left(A_{r}\right) \leq K e^{-\kappa\left(r-r_{0}\right)^{2}}, \forall r>r_{0} .
$$

It is not hard to see that (2.21) can be obtained from (2.22), possibly with degraded constants, i.e., larger $K$ and/or smaller $\kappa$.

The next theorem gives the connection between normal concentration and Talagrand inequality.

Theorem 10. [5, Theorem 3.4.7] Let $(\mathcal{X}, d, \mu)$ be a metric probability space. Then the following two statements are equivalent:

- $\mu$ satisfies $\boldsymbol{T}(\lambda)$.

- $\mu$ has a dimension-free normal concentration with $\kappa=\frac{1}{2 \lambda}$.

\subsection{Conditional Sinkhorn Distance}

To define the conditional Sinkhorn distance, we first give the definition of conditional Wasserstein distance of order $p, p \geq 1$. Given a probability measure $P_{Z}$ and two conditional probability measures $P_{X \mid Z}$ and $P_{Y \mid Z}$ with $P_{X \mid Z=z_{0}}, P_{Y \mid Z=z_{0}} \in \mathcal{P}(\mathcal{Y})$ for all $z$, the Wasserstein distance of order $p$ is defined by

$$
\mathcal{W}_{p}\left(P_{X \mid Z}, P_{Y \mid Z} \mid P_{Z}\right):=\inf _{P \in \Pi\left(\left(P_{X \mid Z}, P_{Y \mid Z} \mid P_{Z}\right)\right)}\left\{\mathbb{E}_{P}\left[d^{p}(X, Y)\right]\right\}^{1 / p},
$$

where

$$
\Pi\left(P_{X \mid Z}, P_{Y \mid Z} \mid P_{Z}\right):=\left\{P_{\bar{X}, \bar{Y} \mid Z} \cdot P_{Z}: P_{\bar{X} \mid Z}=P_{X \mid Z}, P_{\bar{Y} \mid Z}=P_{Y \mid Z}\right\} .
$$

The existence of the optimization problem can be immediately shown if we write (2.23) in another way,

$$
\mathcal{W}_{p}\left(P_{X \mid Z}, P_{Y \mid Z} \mid P_{Z}\right)=\mathbb{E}_{P_{Z}}\left[\mathcal{W}_{p}\left(P_{X \mid Z=z_{0}}, P_{Y \mid Z=z_{0}}\right)\right],
$$

because of the existence of optimal coupling between $P_{X \mid Z=z_{0}}$ and $P_{Y \mid Z=z_{0}}$. From the second definition, we can see that the conditional Wasserstein distance is just a linear form of normal Wasserstein distance, which is trivial.

Similarly, we can add information constraint to conditional Wasserstein 
distance. Conditional Sinkhorn distance is defined by

$\mathcal{W}_{p}\left(P_{X \mid Z}, P_{Y \mid Z} \mid P_{Z} ; R\right):=\inf _{P \in \Pi\left(\left(P_{X \mid Z}, P_{Y \mid Z} \mid P_{Z}\right)\right): I_{P}(X ; Y \mid Z) \leq R}\left\{\mathbb{E}_{P}\left[d^{p}(X, Y)\right]\right\}^{1 / p}$,

where the conditional mutual information is defined by $I_{P}(X ; Y \mid Z):=$ $\int I\left(P_{X \mid Z=z} ; P_{Y \mid Z=z}\right) d P_{Z}(z)$. Because the constraint of conditional mutual information, this problem is not as trivial as (2.23).

Since the constraint of conditional mutual information is a linear form of $I\left(P_{X \mid Z=z} ; P_{Y \mid Z=z}\right)$, the functional and topological properties should be similar to the unconditional one.

\subsection{Entropy Power Inequality and Deconvolution}

EPI [12] states that for all independent continuous random vectors $X$ and $Y$,

$$
N(X+Y) \geq N(X)+N(Y),
$$

where $N(X):=\frac{1}{2 \pi e} e^{\frac{2}{n} h(X)}$ denotes the entropy power of $X$. The equality is achieved when $X$ and $Y$ are Gaussian random vectors with proportional covariance matrices.

Deconvolution is a problem of estimating the distribution $f(x)$ by the observations $Y_{1}, \ldots, Y_{n}$ corrupted by additive noice $Z_{1}, \ldots, Z_{n}$, written as

$$
Y_{i}=X_{i}+Z_{i}
$$

where $X_{i}$ 's are independent and i.i.d distributed in $f(x), Z_{i}$ 's are independent and i.i.d distributed in $h(z) . X_{i}$ 's and $Z_{i}$ 's are mutual independent. Let $g(y)$ be the probability density function of $Y$ that is given by the convolution $g=f * h$. Their entropies can be bounded by EPI immediately.

In our problem, we slightly abuse the concept as simply separating a random vector $Y$ into two independent random vectors $X$ and $Z$. We use this approach to introduce the uncertainty to entropic OT and consequently bound the Sinkhorn distance by EPI. Deconvolution is generally a harder problem than convolution. For instance, log-concave family is convolution stable, i.e., convolution of two log-concave distributions is still log-concave, but we cannot guarantee that deconvolution of two log-concave distributions is still log-concave. A trivial case is the deconvolution of a log-concave distribution by itself is a Dirac function. It should be noted that there are many numerical methods to compute deconvolution, see, e.g., [43, 44, 45]. 
Next, we introduce the kernel-type estimation of the probability density functions. Let $X, Y, Z: \omega \rightarrow \mathbb{R}$. Let $f(x), g(y), h(z)$ be the probability density functions of $X, Y, Z$ respectively. It follows

$$
g(y)=\int_{\mathbb{R}} f(y-u) h(u) d u .
$$

Denote the characteristic functions of $f(x), g(y), h(z)$ by $\phi_{f}(t), \phi_{g}(t)$ and $\phi_{h}(t)$, where $t \in \mathbb{R}$. Then

$$
\phi_{g}(t)=\phi_{f}(t) \phi_{h}(t)
$$

We then introduce a function $K(x)$ used to construct kernel. Let $K(x)$ : $\mathbb{R} \rightarrow \mathbb{R}$ be a real, even, bounded density satisfying $\mathcal{O}\left(\|x\|^{-1-\delta}\right)$ for some $\delta>0$. Denote its Fourier transform by $\phi_{K}(t)$,

$$
\phi_{K}(t)=\int_{\mathbb{R}} e^{i t \cdot x} K(x) d x .
$$

We make following assumptions on $K(x)$ :

- $\left|\phi_{K}(t)\right|>0$, for all $t \in \mathbb{R}$,

- $\phi_{K}(t) / \phi_{K}(t / b) \in L^{1}(\mathbb{R}) \cap L^{\infty}(\mathbb{R}), \quad$ for each $b>0$.

Define a function $W_{b}(x): \mathbb{R} \rightarrow \mathbb{R}$ with $b>0$, by

$$
W_{b}(x)=\frac{1}{2 \pi} \int_{\mathbb{R}} e^{i t \cdot x}\left[\phi_{K}(t) / \phi_{h}(t / b)\right] d t .
$$

Let $\left\{b_{n}\right\}_{n=1}^{\infty}$ be a sequence of positive numbers such that $b_{n} \rightarrow 0$ as $n \rightarrow n$. Given the observations $\left\{Y_{i}\right\}_{i=1}^{n}$, the estimator $\hat{f}_{n}(x)$ have the representation

$$
\hat{f}_{n}(x)=\frac{1}{n b_{n}} \sum_{j=1}^{n} W_{b_{n}}\left[\left(Y_{j}-x\right) / b_{n}\right]
$$

We can see that $\hat{f}_{n}(x)$ has the form of a kernel density estimator. It is also clear that (2.26) is not always integrable. It makes the choice of the function $K(x)$ important and is the reason we apply kernel method in Chapter 3. When $W_{b_{n}} \in L^{1}(\mathbb{R})$, by (2.26) and the inversion formula, we have

$$
\phi_{K}(t) / \phi_{h}(t / b)=\int_{\mathbb{R}} W_{b_{n}}(x) e^{-i t \cdot x} d x .
$$


24 | Background

We note that there is an alternative expression for the estimator,

$$
\hat{f}_{n}(x)=\frac{1}{2 \pi} \int_{\mathbb{R}} e^{-i t \cdot x} \hat{\phi}_{g, n}(t) \frac{\phi_{K}\left(b_{n} t\right)}{\phi_{h}(t)} d t
$$

where

$$
\hat{\phi}_{g, n}(t)=\frac{1}{n} \sum_{j=1}^{n} e^{i t \cdot Y_{j}} .
$$

The asymptotic behavior of $\hat{f}_{n}(x)$ is also studied in related literature. The following theorem points out that $\hat{f}_{n}(x)$ is a consistent estimator.

Theorem 11. For almost all $x \in \mathbb{R}$ we have

$$
\mathbb{E}\left[\hat{f}_{n}(x)\right] \rightarrow f(x), \quad \text { as } \quad n \rightarrow \infty
$$

Some other asymptotic expressions and bounds of quadratic-mean estimation error can be obtained for the estimator $\hat{f}_{n}(x)$, cf., [43]. [45] studies the multivariate kernel-type deconvolution problem for stationary random processes, which may be adapted to independent random vector case. 


\section{Chapter 3}

\section{Main Results}

In this chapter, we present our main results.

\subsection{Theoretical Results}

In this section, we derive our main theoretical results. First, we give a new HWI-type inequality.

Theorem 12 (HWI-type Inequality). Let $\mathcal{X}=\mathcal{Y}=\mathbb{R}^{n}$. Let $\mu$ be a probability measure with density $e^{-V(x)}$ with $\lambda>0$, where $V: \mathbb{R}^{n} \rightarrow \mathbb{R}$ is $C^{2}$ continuous, $D^{2} V \geq \lambda I_{n}$. Let $P_{X}, P_{Y}$ be two probability measures on $\mathbb{R}^{n}, P_{X}, P_{Y} \ll \mu$. For any independent $Y_{1}, Y_{2}$ satisfying $Y_{1}+Y_{2}=Y, \mathbb{E}\left[Y_{2}\right]=0$ and $h(Y)-$ $h\left(Y_{2}\right) \leq R$, we have

$$
\begin{aligned}
& \frac{\lambda}{2} \mathcal{W}_{2}^{2}\left(P_{X}, P_{Y} ; R\right) \leq \mathbb{E}[V(Y)]-\mathbb{E}[V(X)]+n \\
& \quad-n e^{\frac{1}{n}\left(h\left(Y_{1}\right)-h(X)\right)}+\mathcal{W}_{2}\left(P_{X}, P_{Y_{1}}\right) \sqrt{I\left(P_{X} \mid \mu\right)}
\end{aligned}
$$

Proof. See Appendix A.1.

The next result gives a new Talagrand-type inequality.

Theorem 13 (Talagrand-type Inequality). Let $\mathcal{X}=\mathcal{Y}=\mathbb{R}^{n}$. Let $d P_{X}=$ $e^{-V(x)} d x$, where $V: \mathbb{R}^{n} \rightarrow \mathbb{R}$ is $C^{2}$ continuous, $D^{2} V \geq \lambda I_{n}$ with $\lambda>0$, $P_{Y} \ll P_{X}$. We have

$$
\begin{aligned}
\frac{\lambda}{2} \mathcal{W}_{2}^{2}\left(P_{X}, P_{Y} ; R\right) \leq & \mathbb{E}[V(Y)]-\mathbb{E}[V(X)]+n \\
& -n C\left(P_{Y}, R\right) e^{\frac{1}{n}(h(Y)-h(X))},
\end{aligned}
$$


where $C\left(P_{Y}, R\right) \in[0,1]$ is a numerical term for the given $P_{Y}$ and $R \geq 0$.

Proof. Let $d P_{X}=e^{-V}$ in (3.1). In such case, we have $I\left(P_{X} \mid \mu\right)=0$ from the definition of relative fisher information. Take $C\left(P_{Y}, R\right)=e^{\frac{1}{n}\left(h\left(Y_{1}\right)-h(Y)\right)}$, then (3.2) is proved from (3.1).

Next, we state some technical remarks on Theorem 13.

Remark (On Theorem 13). The equality of (A.1) holds when $P_{X}$ is isotropic Gaussian, i.e., $P_{X} \sim \mathcal{N}\left(\mu, \sigma^{2} I_{n}\right)$ for some $\mu \in \mathbb{R}^{n}$ and $\sigma>0$. The equality in Lemma 20 holds when $\nabla \varphi$ is affine and $D^{2} \varphi$ has identical eigenvalues, i.e., $\nabla \varphi=k \cdot I d, k \in \mathbb{R}$, see [4, Lemma 2.6]. From [46, Theorem 1] we know that the linear combination $Y=Y_{1}+Y_{2}$ in Theorem 12 is the optimizer for entropic OT when $X$ and $Y$ are isotropic Gaussian. In such case, the equality of (3.2) holds and $C(\cdot, R)=\sqrt{1-e^{-\frac{2}{n} R}}$.

Remark (On the numerical term $C(\cdot, \cdot)$ ). We observe that $e^{\frac{1}{n} h\left(Y_{1}\right)}$ has the form of a square root of entropy power. Using EPI and the fact that $N(\cdot) \geq 0$, we have

$$
N(Y) \geq N\left(Y_{1}\right)+N\left(Y_{2}\right) \geq N\left(Y_{1}\right) .
$$

Therefore $C=e^{\frac{1}{n}\left(h\left(Y_{1}\right)-h(Y)\right)}=\sqrt{N\left(Y_{1}\right) / N(Y)} \in[0,1]$. When $R=0$, $Y=Y_{2}$, the density of $Y_{1}$ is $\delta(x)$. It means that $e^{\frac{1}{n} h\left(Y_{1}\right)}=0$, hence $C=0$. When $R=1, Y=Y_{1}, e^{\frac{1}{n} h\left(Y_{1}\right)}=e^{\frac{1}{n} h(Y)}$, hence $C=1$. Now assume that we have $R^{\prime}<R$. Then $\Pi\left(P_{X}, P_{Y} ; R^{\prime}\right) \subset \Pi\left(P_{X}, P_{Y} ; R\right)$ and the minimization problem $\left(\mathcal{P}_{R}\right)$ directly leads to $\mathcal{W}_{2}\left(P_{X}, P_{Y} ; R^{\prime}\right) \geq \mathcal{W}_{2}\left(P_{X}, P_{Y} ; R\right)$. Note that $\mathcal{W}_{2}\left(P_{X}, P_{Y} ; R\right)$ can be also bounded by $C\left(P_{Y}, R^{\prime}\right)$. Hence $C\left(P_{Y}, R^{\prime}\right) \leq$ $C\left(P_{Y}, R\right)$, i.e., $C(\cdot, \cdot)$ is a monotonic non-decreasing function with respect to $R$. Moreover, $C(\cdot, 0)=0, C(\cdot,+\infty)=1$ for all $P_{Y}$.

We note that for particular distributions, we may have explicit expression of $C(\cdot, \cdot)$. When $P_{Y}$ is Gaussian, we can always take the linear combination $Y=Y_{1}+Y_{2}$, where $Y_{1}$ and $Y_{2}$ are independent Gaussian and have proportional covariance matrices. In such case, EPI is saturated as follows,

$$
\begin{aligned}
e^{\frac{2}{n} h\left(Y_{1}\right)} & =e^{\frac{2}{n} h(Y)}-e^{\frac{2}{n} h\left(Y_{2}\right)} \\
& =\left(1-e^{-\frac{2}{n} R}\right) e^{\frac{2}{n} h(Y)} .
\end{aligned}
$$

As a result, we have $C\left(P_{Y}, R\right)=e^{\frac{1}{n}\left(h\left(Y_{1}\right)-h(Y)\right)}=\sqrt{1-e^{-\frac{2}{n} R}}$. For Cauchy distribution Cauchy $\left(x_{0}, \gamma\right)$, its differential entropy is $\log (4 \pi \gamma)$. The summation of independent Cauchy random variables $\sum_{i}^{n}$ Cauchy $\left(x_{i}, \gamma_{i}\right) \sim$ $\operatorname{Cauchy}\left(\sum_{i}^{n} x_{i}, \sum_{i}^{n} \gamma_{i}\right)$. When $Y$ is i.i.d. Cauchy, i.e., $(Y)_{i} \sim \operatorname{Cauchy}\left(x_{0}, \gamma\right)$, we take $\left(Y_{1}\right)_{i} \sim \operatorname{Cauchy}\left(x_{0}, \frac{1}{4 \pi} e^{\frac{1}{n} h(Y)} \cdot\left(1-e^{-\frac{R}{n}}\right)\right)$ and $\left(Y_{2}\right)_{i} \sim \operatorname{Cauchy}\left(0, \frac{1}{4 \pi} e^{\frac{1}{n} h(Y)}\right.$. 
$\left.e^{-\frac{R}{n}}\right)$. We can see this linear combination satisfies our assumption $h(Y)-$ $h\left(Y_{2}\right) \leq R$ and $C\left(P_{Y}, R\right)=e^{\frac{1}{n}\left(h\left(Y_{1}\right)-h(Y)\right)}=1-e^{-\frac{R}{n}}$.

Note that the linear combination $Y=Y_{1}+Y_{2}$ is not unique, according to the assumption of Theorem 12. Consequently, it leads to the non-uniqueness of $C(\cdot, \cdot)$. In order to obtain the tightest bound in (3.2), the optimal $C^{*}\left(P_{Y}, R\right)=$ $\sup e^{\frac{1}{n}\left(h\left(Y_{1}\right)-h(Y)\right)}$ subject to $Y_{1}+Y_{2}=Y$ and $h(Y)-h\left(Y_{2}\right) \leq R$. To look into this optimization problem, we introduce Courtade's reverse EPI [47, Corollary 1] as follows. If we have independent $X$ and $Y$ with finite second moments and choose $\theta$ to satisfy $\theta /(1-\theta)=N(Y) / N(X)$, then

$$
N(X+Y) \leq(N(X)+N(Y))(\theta p(X)+(1-\theta) p(Y)),
$$

where $p(X):=\frac{1}{n} N(X) J(X) \geq 1$ is Stam defect. $p(X)$ is affine invariant, i.e. $p(X)=p(t X), t>0$ because $t^{2} N(X)=N(t X)$ and $J(X)=t^{2} J(X)$. The equality $p(X)=1$ holds only if $X$ is Gaussian. In our case, $\theta=$ $N\left(Y_{1}\right) /\left(N\left(Y_{1}\right)+N\left(Y_{2}\right)\right)$. When $\theta \rightarrow 1$, (3.3) becomes

$$
N(Y) \lesssim\left(N\left(Y_{1}\right)+N\left(Y_{2}\right)\right) \cdot p\left(Y_{2}\right)
$$

It means the saturation of EPI is controlled by $p\left(Y_{2}\right)$ when the noise $Y_{2}$ is small, i.e., $R$ is large. In this case, $C^{*}\left(P_{Y}, R\right) \approx \sqrt{1-e^{-\frac{2}{n} R}}$ if we let $Y_{2}$ close to Gaussian, i.e., $p\left(Y_{2}\right)=1$. On the other hand, when $\theta \rightarrow 0$, EPI can also be saturated if we let $Y_{1}$ close to Gaussian.

In Fig. 3.1, we illustrate numerical simulations of $C(\cdot, \cdot)$ for one-dimensional case. For general distributions beyond Gaussian and i.i.d. Cauchy, we approximate $C(\cdot, \cdot)$ using kernel methods of deconvolution, see, e.g., [43, 44]. Our strategy of deconvolution in Fig. 3.1 is to let $Y_{2}=t Y^{\prime}$, where $Y^{\prime}$ is a copy of $Y$ and $t \in[0,1]$. Gaussian mixture is an exception for this strategy because its spectrum would be not integrable. Instead we let $Y_{2}$ to be Gaussian for Gaussian mixture. We note this strategy is mostly not optimal and the optimal way to maximize the entropy power above is still an open question.

The following corollary is immediate from Theorem 13.

Corollary 13.1. Wasserstein distance is bounded by

$$
\frac{\lambda}{2} \mathcal{W}_{2}^{2}\left(P_{X}, P_{Y}\right) \leq \mathbb{E}[V(Y)]-\mathbb{E}[V(X)]+n-n e^{\frac{1}{n}(h(Y)-h(X))} .
$$

Proof. This is immediate from Theorem 13 when $R \rightarrow \infty$. 


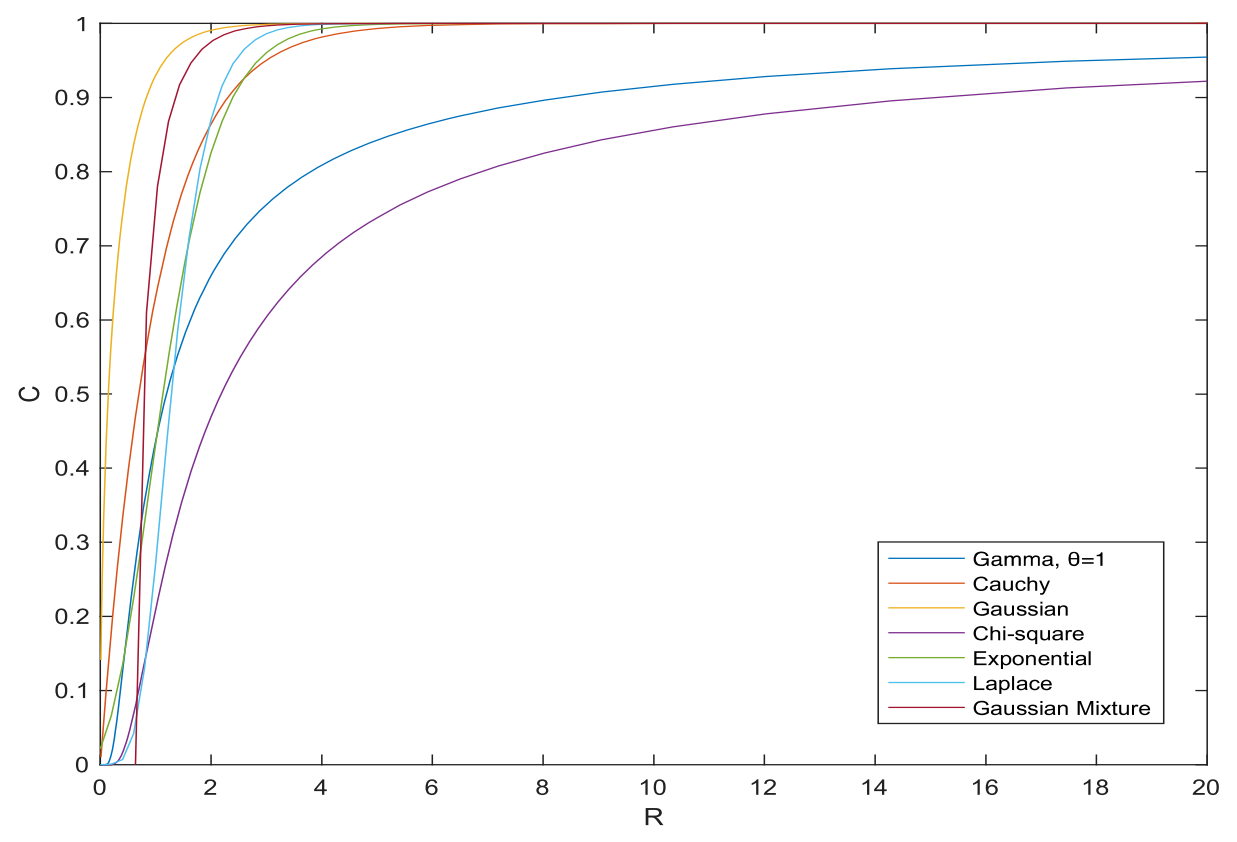

Figure 3.1 - Plot of the numerical term $\mathrm{C}$ subject to the information constraint $R$ evaluated with respect to different distributions for the one dimensional case.

Remark. We note that (3.4) is equivalent to Bolley's dimensional Talagrand inequality (2.17) and it is tighter than the classical Talagrand inequality (2.15). Notice under our assumptions, $h(X)=\mathbb{E}[V(X)]$ and $D\left(P_{Y} \| P_{X}\right)=$ $\mathbb{E}[V(Y)]-h(Y)$ because $d P_{X}=e^{-V(x)} d x$. Clearly, by substituting these expressions to the last term of (3.4) we obtain (2.17). Since $e^{\mu} \geq 1+\mu,(2.17)$ is, in general, tighter than the classical Talagrand inequality (2.15), i.e., R.H.S. of $(2.17) \leq$ R.H.S. of (2.15). The equality holds if and only if $h(Y)=h(X)$.

We notice that $C$ is the only difference between (2.17) and (3.2), from Remark 3.1. Therefore, we can immediately get a result of measure concentration following [4, Corollary 2.4]. Here we state the result of measure concentration obtained from (3.2).

Corollary 13.2. Let $d \mu=e^{-V}$, where $V: \mathbb{R}^{n} \rightarrow \mathbb{R}$ is $C^{2}$ continuous, $D^{2} V \geq$ $\lambda I_{n}$ with $\lambda>0$. Let $A \subset \mathbb{R}^{n}, A_{r}:=\left\{x \in \mathbb{R}^{n} \mid \forall y \in A,\|x-y\|>r\right\}$ for $r \geq 0$ and $c_{A}:=\sqrt{2 \lambda^{-1} \log (1 / \mu(A))}$. Then for $r \geq c_{A}$,

$$
\mu\left(A_{r}\right) \leq C^{-n} \cdot e^{-\frac{\lambda}{2}\left(r-c_{A}\right)^{2}} .
$$

Furthermore, we can proof that $C^{n}$ decreases with the increment of dimension under a natural assumption. 
Proof. See Appendix A.2.

Remark. The new inequality from Sinkhorn distance still implies a normal concentration. The difference is that it is dimension dependent, compared to the dimension-free one in [4]. Note that $C^{n}$ decreases with the increment of dimension. Hence, the increment of dimension leads to a slower concentration, i.e., a looser bound in (3.5).

The next theorem is another Talagrand-type inequality. Compared to Theorem 3, the following theorem is a bound obtained using a term related to the saturation of $P_{X}$ instead of the saturation of $P_{Y}$ that was used in Theorem 13.

Theorem 14. Let $\mathcal{X}=\mathcal{Y}=\mathbb{R}^{n}$. Without loss of generality, let $X$ be a zero-mean random vector with density $e^{-V(x)}$, where $V: \mathbb{R}^{n} \rightarrow \mathbb{R}$ is $C^{2}$ continuous, $D^{2} V \geq \lambda I_{n}$ with $\lambda>0, P_{Y} \ll P_{X}$. Then we have

$$
\begin{aligned}
\frac{\lambda}{2} \mathcal{W}_{2}^{2}\left(P_{X}, P_{Y} ; R\right) & \leq \mathbb{E}[V(Y)]-\mathbb{E}[V(X)]+n \\
& -n C_{x}\left(P_{X}, R\right) e^{\frac{1}{n}(h(Y)-h(X))}+\epsilon,
\end{aligned}
$$

where $\epsilon$ is a term related to the linearity of $V$.

Proof. See Appendix A.3.

We make the following technical comments on Theorem 14 .

Remark (On Theorem 14). Similar to $C\left(P_{Y}, R\right), C_{x}\left(P_{X}, R\right)$ is also related to the saturation of EPI, as shown in the proof. However, (3.6) is less natural than (3.2) because of the extra term $\epsilon$. When $\nabla V$ is nearly linear, $\epsilon$ should be small. When $\nabla V$ is far from linear, $\epsilon$ is unknown.

Remark. When $\nabla V$ is linear, $\epsilon$ is zero and $C_{x}(\cdot, R)=\sqrt{1-e^{-\frac{2}{n} R}}$, as simply taking $t=\sqrt{1-e^{-\frac{2}{n} R}}$ in the proof. In such case, (3.6) recovers (2.18) by taking $X$ as a standard Gaussian, i.e. $V(x)=\|x\|^{2} / 2+k$, where $k$ is a normalization factor. Substitute $V$ and times 2 on both sides of (3.6), we have

$$
\begin{aligned}
\mathcal{W}_{2}^{2}(X, Y ; R) & \leq \mathbb{E}\left[\|Y\|^{2}\right]-\mathbb{E}\left[\|X\|^{2}\right]+2 n-2 n \sqrt{1-e^{-\frac{2}{n} R}} e^{\frac{1}{n}(h(Y)-h(X))} \\
& =\mathbb{E}\left[\|Y\|^{2}\right]+n-2 n \sqrt{\frac{1}{2 \pi e}\left(1-e^{-\frac{2}{n} R}\right) e^{\frac{1}{n} h(Y)}}
\end{aligned}
$$

Theorem 15 (Talagrand-type Bound for Conditional Sinkhorn Distance). For $\mathcal{X}=\mathcal{Y}=\mathbb{R}^{n}$, given a probability measure $P_{Z}$ and two conditional probability 
measures $P_{X \mid Z}$ and $P_{Y \mid Z}$, where the probability density $d P_{X \mid Z=z_{0}}=d P_{X}=$ $e^{-V(x)} d x, V: \mathbb{R}^{n} \rightarrow \mathbb{R}$ is $C^{2}$ continuous, $D^{2} V \geq \lambda I_{n}$ with $\lambda>0, P_{Y \mid Z=z_{0}} \ll$ $P_{X}$, we have

$$
\begin{aligned}
\frac{\lambda}{2} \mathcal{W}_{2}^{2}\left(P_{X}, P_{Y} \mid P_{Z} ; R\right) \leq \mathbb{E}[V(Y \mid Z)] & -\mathbb{E}[V(X)]+n \\
& -n C^{\prime}\left(P_{Y \mid Z}, R\right) e^{\frac{1}{n}(h(Y \mid Z)-h(X))},
\end{aligned}
$$

where $C^{\prime}\left(P_{Y \mid Z}, R\right)$ is also a numerical term. A direct observation is that $C^{\prime}\left(P_{Y \mid Z}, R\right)$ can take $\inf _{z_{0}} C\left(P_{Y \mid Z=z_{0}}, R\right)$.

Proof. See Appendix A.4.

\subsection{Physical Interpretation of Our Results}

McCann's interpolation [25, Chapter 5] claims OT problem can be understood as a process from an initial measure $\mu_{0}$ to a final measure $\mu_{1}$ along the geodesic on a functional space. The proofs of Talagrand inequalities use techniques like heat semigroup and infinitesimal displacement convexity, see, e.g., Lemma 20 in Appendix A.5. We give a brief intuition behind these techniques. It is well known that KL-divergence is convex along the geodesic between two given measures $\mu_{0}$ and $\mu_{1}$. We can give an estimate of the KL-divergence using the infinitesimal displacement at $\mu_{0}$, which can be simply understood as the velocity at $\mu_{0}$ along its geodesic. In Talagrand inequality, $\mu_{0}$ is Gaussian or strongly log-concave, which leads to a simple form of estimate.

When considering Sinkhorn distance, we need to introduce a stochastic kernel (defined in Appendix A.5.5) during the transport because of the uncertainty brought by the constraint of mutual information. Practically, we construct a linear combination of two independent random vectors, i.e., find $\mu^{1}$ and $\mu^{2}$ satisfying $d \mu_{i}=d \mu^{1} * d \mu^{2}, i \in\{0,1\}$, following the proof in [11]. It is shown in Theorem 14 that if we separate $\mu_{0}$, the estimate from the infinitesimal displacement at $\mu_{0}$ becomes complicated and hard to generalize far from Gaussian distribution. On the other hand, we separate $\mu_{1}$ and obtain a simpler form in Theorem 13. Theorems 13, 14 do not recover each other because they correspond to two different processes behind the proofs. 


\subsection{Numerical Simulations}

In this section, we give numerical simulations for Theorem 13 and 14 in Chapter 3. We use the Sinkhorn algorithm from POT library [48]. Because of the precision of the library, a limited range of the rate $R$ is simulated in the following experimental results. We plot the results from (13), (14) by orange curves and the simulations by Sinkhorn algorithm by blue curves.

In Figure 3.2a, we give the result of joint Gaussian marginals, which is the scenario when the identity in Theorem 13 holds. From the figure, we can see the simulation is slightly greater than the bound. A reasonable explanation is that we terminate the Sinkhorn iteration when the pair $\left(\boldsymbol{a}^{(l)}, \boldsymbol{b}^{(l)}\right)$ converge to some rate. Therefore, there is still a small difference between the optimizer $\left(\boldsymbol{a}^{*}, \boldsymbol{b}^{*}\right)$ and $\left(\boldsymbol{a}^{(l)}, \boldsymbol{b}^{(l)}\right)$. On the other hand, it also implies that this algorithm has a reasonable precision. We also give other two simulations for bound (14) in Figure 3.2b and 3.2c.

For Theorem 14, we mainly simulate the case when one side of the distributions is Gaussian, in Figure 3.3, because the term $\epsilon$ is hard. We use the fixed $V=\frac{x^{2}}{2}$ and numerical term $C=\sqrt{1-R^{\frac{2}{n}}}$.

In both bounds, we avoid the unknown factors and find several interesting behaviors of the bound. We observe that the original Talagrand inequality (2.15) is not tight when $P_{Y}$ is not absolutely continuous with respect to $P_{X}$. We give such simulations of the case when $P_{Y}$ tends to not absolutely continuous to $P_{X}$, as illustrated in Figure 3.4. It can be seen from Figure 3.5 that the bound becomes looser because the difference of $\mathbb{E}[V]$ between two random variables is exacerbated with the higher moments. Moreover, the difference is big no matter what value $R$ is, which implies that (2.15) and (2.17) are also loose with the discontinuity. The bound can be worse if we increase the discontinuity, as shown in Figure 3.6. By simply changing the sides of distributions $P_{X}$ and $P_{Y}$, the discontinuity disappears and the bound becomes tight, in Figure $3.5 \mathrm{~b}$ and $3.6 \mathrm{~b}$. The non-absolutely continuity, which is also called non-overlapping in machine learning community, is the reason of mode collapse in GAN. Wasserstein distance solves this problem [8].

We can also compare Figure 3.3a and 3.2b. We observe that $C=$ $\sqrt{1-R^{\frac{2}{n}}}$ gives a better description of Sinkhorn distance than $C=1-R^{\frac{1}{n}}$, which also consists with our previous discussion that the independent linear combination of Cauchy random variables is not the optimal deconvolution. Actually, even if the distribution is not Gaussian, $C=\sqrt{1-R^{\frac{2}{n}}}$ appears to be true for our bound, according to the numerical simulations we did. This result is consistent with our discussion in Remark 3.1. 
Furthermore, the tightness of the bound seems related to Fisher information, which quantitatively describes the regularity of a measure. For example, Cauchy distribution and Laplace distribution are similar to Gaussian. Thus, they show a tight bound in Figure 3.3a and 3.3d. On the other hand, Gaussian mixture and exponential distribution are relatively far from Gaussian. So Figure $3.3 \mathrm{f}$ and $3.3 \mathrm{c}$ show a looser bound.

In Figure 3.7, we plot the dimensionality of Sinkhorn distance for isotropic Gaussians. It can be seen that the information constraint causes more smoothing on higher dimension for the original Wasserstein distance, which is consistent with Corollary 13.2. It provides a wider boundary for decision for small disturbance on high dimension for classification and may be one of the explanation that Sinkhorn distance outperform other metrics in [10]. 


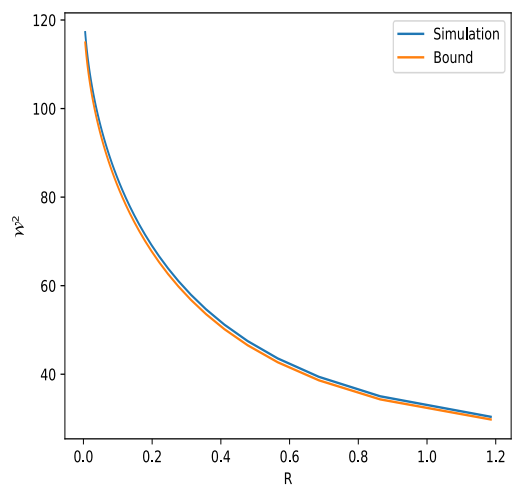

(a) $d P_{X} \sim \mathcal{N}\left(0, \frac{1}{25}\right)$ and $d P_{Y} \sim$ $\mathcal{N}\left(0, \frac{1}{100}\right)$.

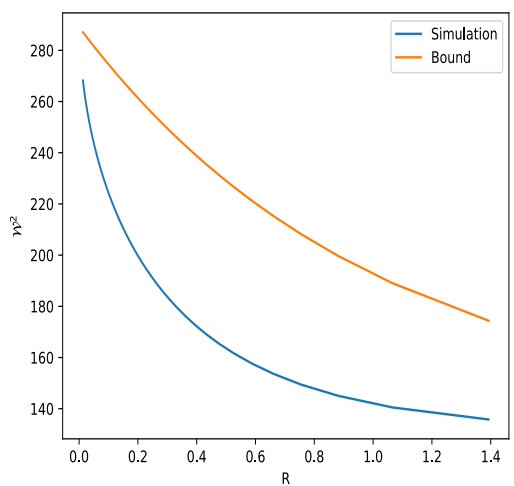

(b) $d P_{X} \sim \mathcal{N}\left(0, \frac{1}{25}\right)$ and $d P_{Y} \sim$ Cauchy $(0,10)$.

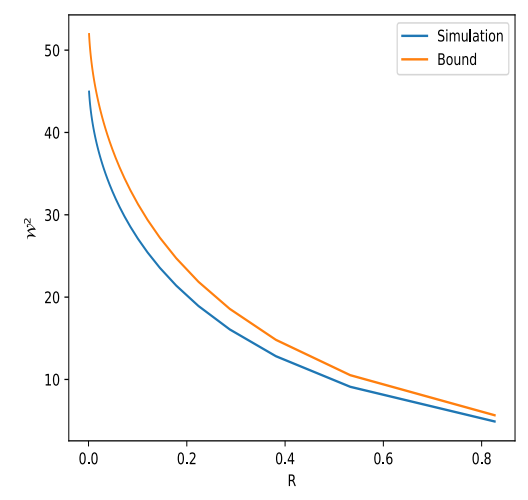

(c) $d P_{X}=e^{-V}, V=(x / 5)^{2} / 2+$ $|x / 10|+e^{-|x / 10|}+k, k \in \mathbb{R}$ and $d P_{Y} \sim \mathcal{N}\left(0, \frac{1}{25}\right)$

Figure 3.2 - Numerical simulations and bounds (3.2) subject to different $R$. 


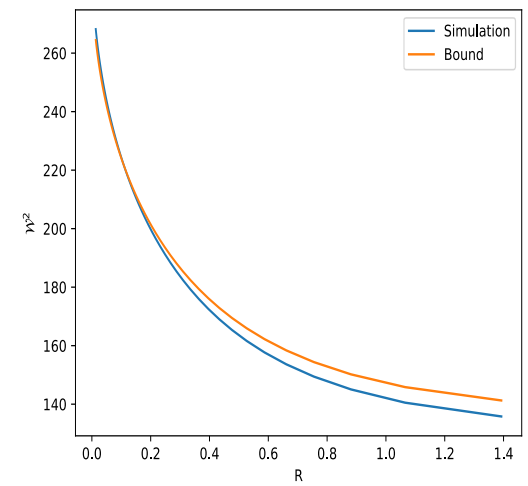

(a) $d P_{X} \sim \mathcal{N}\left(0, \frac{1}{25}\right)$ and $d P_{Y} \sim$ Cauchy $(0,10)$.

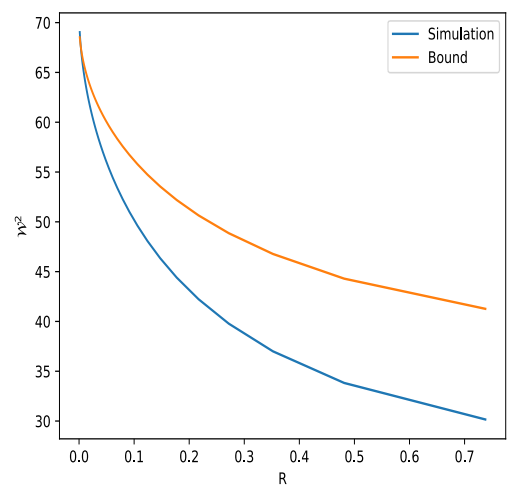

(c) $d P_{X} \sim \mathcal{N}\left(0, \frac{1}{25}\right)$ and $d P_{Y} \sim$ $\operatorname{Exp}(0.2)$.

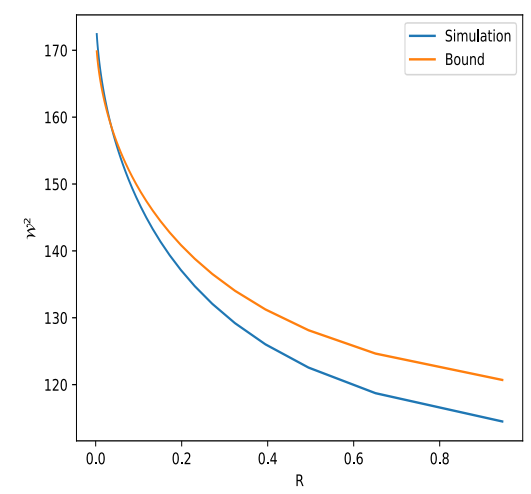

(e) $d P_{X} \sim \mathcal{N}\left(0, \frac{1}{25}\right)$ and $d P_{Y}$ is Gamma distribution with $\alpha=2$ and $\beta=0.2$.

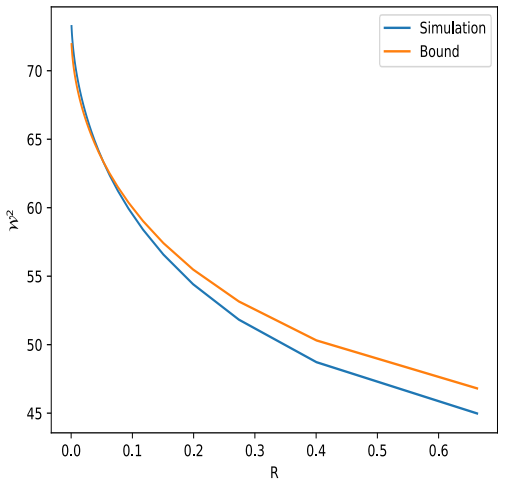

(b) $d P_{X} \sim \mathcal{N}\left(0, \frac{1}{25}\right)$ and $d P_{Y} \sim$ $\chi^{2}(6)$.

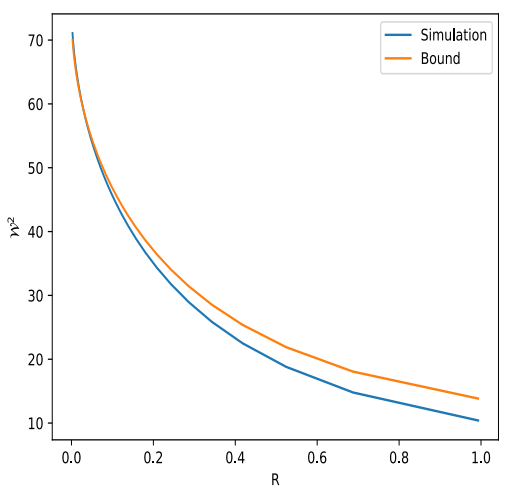

(d) $d P_{X} \sim \mathcal{N}\left(0, \frac{1}{25}\right)$ and $d P_{Y} \sim$ Laplace $(0,5)$.

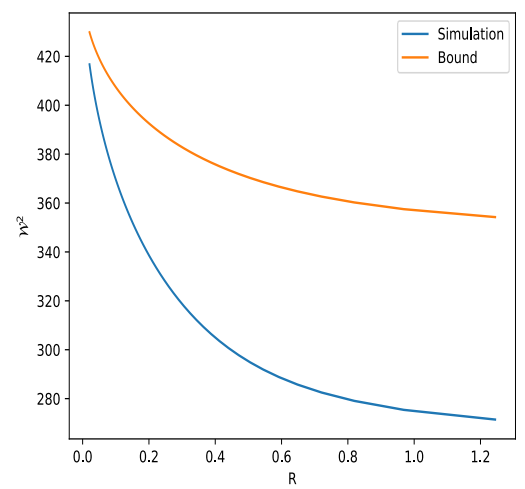

(f) $d P_{X} \sim \mathcal{N}\left(0, \frac{1}{25}\right)$ and $d P_{Y}=$ $\frac{1}{2} \mathcal{N}\left(-20, \frac{1}{25}\right)+\frac{1}{2} \mathcal{N}\left(20, \frac{1}{25}\right)$.

Figure 3.3 - Numerical simulations and bounds (3.6) subject to different $R$. 


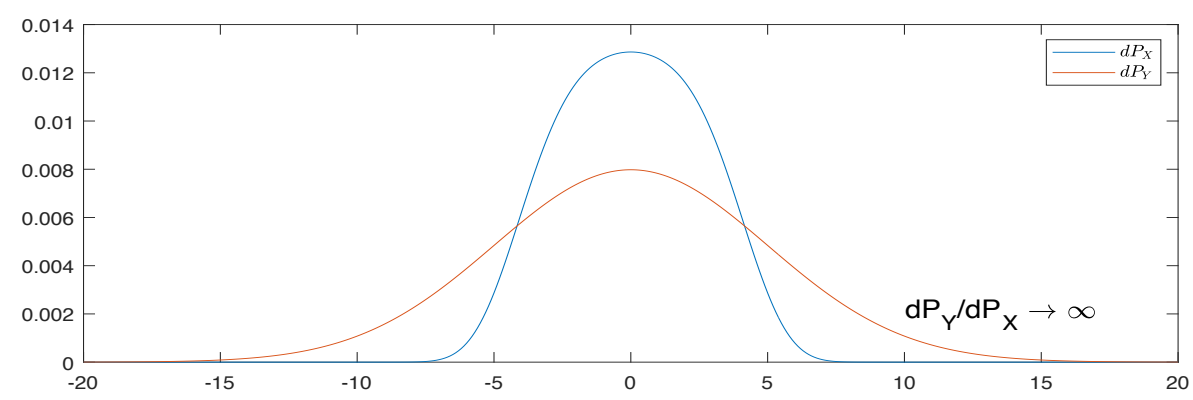

Figure 3.4 - Probability densities of $d P_{X}=e^{-V}, V=(x / 5)^{2} / 2+(x / 5)^{4}+$ $k, k \in \mathbb{R}$ and $d P_{Y} \sim \mathcal{N}\left(0, \frac{1}{25}\right)$.

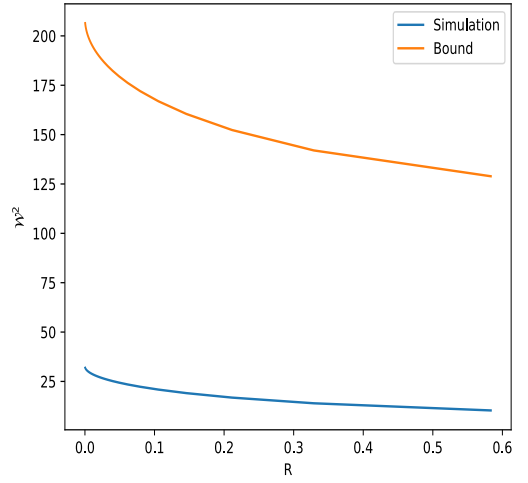

(a) Bound (3.2) with $d P_{X}=d \mu$ and $d P_{Y}=d \nu$.

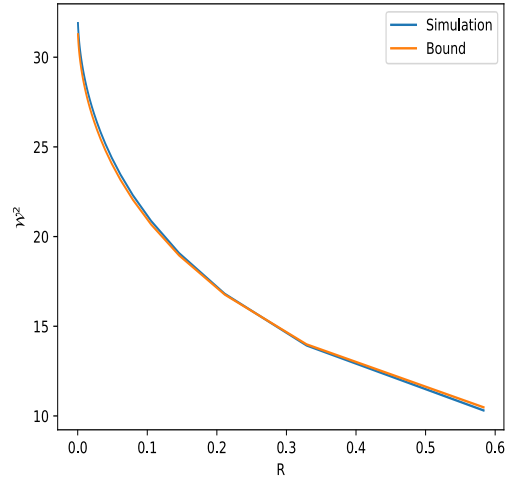

(b) Bound (3.6) with $d P_{X}=d \nu$ and $d P_{Y}=d \mu$.

Figure 3.5 - Numerical simulations and bounds subject to different $R$, for $d \mu=$ $e^{-V}, V=(x / 5)^{2} / 2+(x / 5)^{4}+k, k \in \mathbb{R}$ and $d \mu \sim \mathcal{N}\left(0, \frac{1}{25}\right)$. 


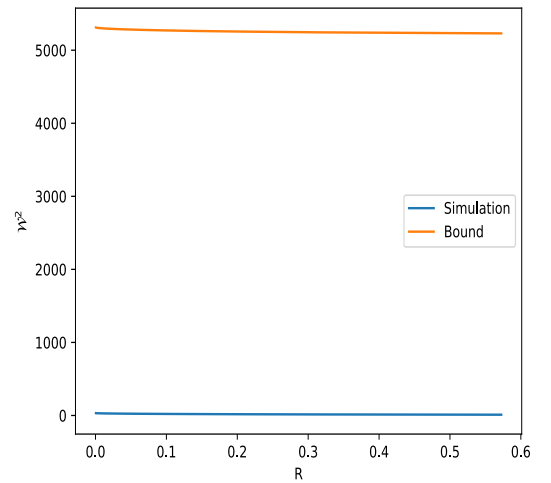

(a) Bound (3.2) with $d P_{X}=d \mu$ and $d P_{Y}=d \nu$.

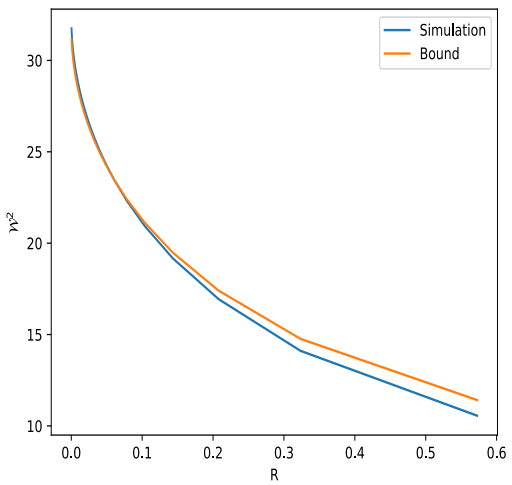

(b) Bound (3.6) with $d P_{X}=d \nu$ and $d P_{Y}=d \mu$.

Figure 3.6 - Numerical simulations and bounds subject to different $R$, for $d \mu=$ $e^{-V}, V=(x / 5)^{2} / 2+(x / 5)^{8}+k, k \in \mathbb{R}$ and $d \mu \sim \mathcal{N}\left(0, \frac{1}{25}\right)$.

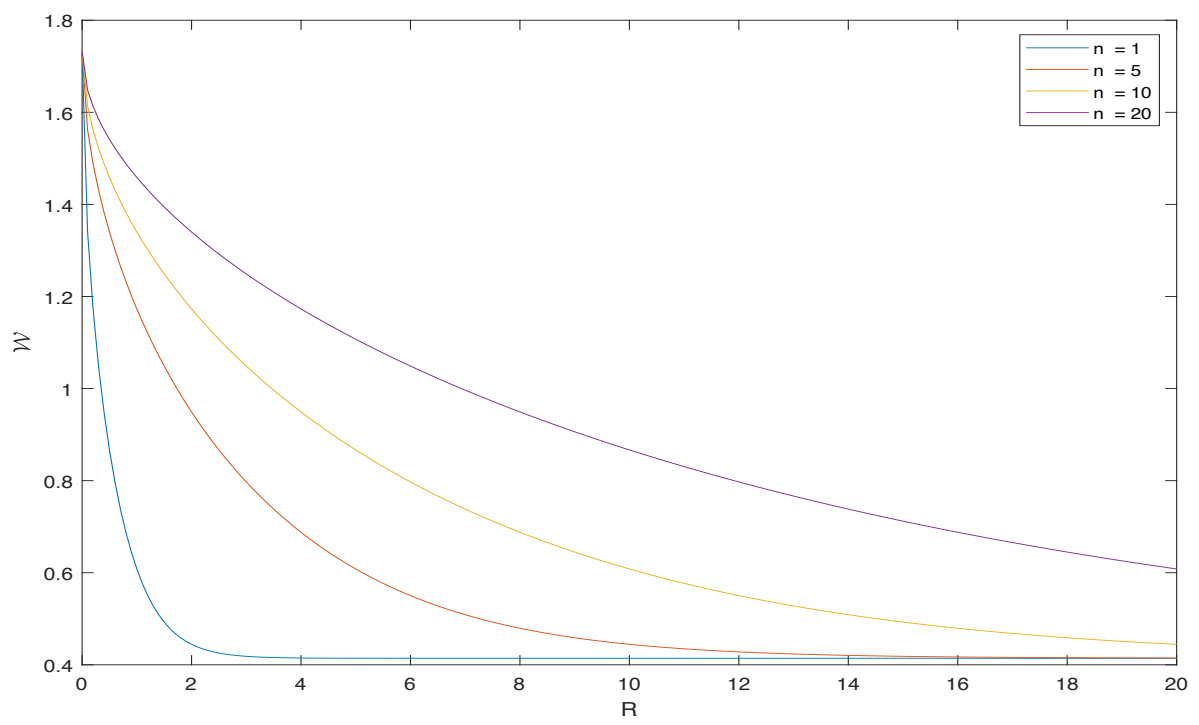

Figure 3.7 - Sinkhorn distances between isotropic Gaussians on different dimensions. 


\section{Chapter 4}

\section{Conclusions and Future work}

\subsection{Conclusions}

In this thesis, we study the properties of Sinkhorn distance defined by Cuturi. Our main result is a new Talagrand-type inequality, which contains a numerical term related to the saturation of EPI. Because there is no more deterministic mapping between two random vectors in entropic OT, finding an estimate of Sinkhorn distance is harder than the original Wasserstein distance. A stochastic kernel increases the dimension of optimization problem and also increases the difficulty for the estimate of the quadratic distance. In our results, the uncertainty from the stochastic kernel is captured by EPI.

We show that the Sinkhorn distance of two random vectors can be upper bounded by their moments and differential entropy. Also, both sides of (3.2) both go to zero when the two random vectors are identical. Therefore, R.H.S. of (3.2) has a stronger convergence than Sinkhorn distance. Interestingly, the numerical term caused by the information constraint is before the term related to the entropy powers. It means that the effect of information constraint is only associated with the randomness of the two random vectors instead of their positions. It consequently leads to a dimensional concentration of measure. Because the measure concentration inequality is derived by Sinkhorn distance, it may further implies the sample complexity to train a deep learning model below a certain rate of error using Sinkhorn distance. Mode collapse on high dimensional noise may also be another consequence for this phenomenon.

One topic throughout this work is the regularity of a probability measure. We may simply regard that the regularity can be described by Fisher information. Without the assumption of strongly log-concavity, it requires an extra term of relative Fisher information in Theorem 12. EPI and deconvolution are also 
concerned with Fisher information. Moreover, a measure is not so regular if it is supported on a set with finite volume with respect to Lebesgue measure, because, according to heat flow, a mass should diffuse continuously. However, for an artificial intelligence, it is impossible to study a sample infinitely far from its expectation. More technically, ReLU is impossible to fit the tail of a Gaussian measure. Thus, KL-divergence, which was originally used in the context of physics, gives a large value and consequently leads to mode collapse in GAN training. This problem also happens in our numerical simulation. In this situation, Wasserstein distance is shown to be a better metric for two probability measures to train a deep neural network [8].

Another interesting topic is the dimensionality of Sinkhorn distance. From the measure concentration inequality (3.5) and Figure 3.7, we can see Sinkhorn distance is more sensitive to the smoothing of information constraint on high dimension. We know that, in machine learning, samples are usually embeded in a low dimension manifold with the disturbance of a high dimension noise and we want to recover the low-dimensional structure $[49,50]$. Sinkhorn distance may have the potential to adapt to low-dimensional structure and be computationally tractable via SGD. Moreover, the dimensionality of Sinkhorn distance can be one of the explanations that it outperforms other metrics in the experimental results of [10].

\subsection{Future work}

\subsubsection{Entropy Bound}

As an important step in our main result, we want to find an estimate for the optimizer $C^{*}=\sup e^{\frac{1}{n}\left(h\left(Y_{1}\right)-h(Y)\right)}$ subject to $Y_{1}+Y_{2}=Y$ and $h(Y)-h\left(Y_{2}\right) \leq$ $R$. Particularly, this problem is equivalent to find a lower bound for the differential entropy $h\left(Y_{1}\right)$.

It is well known that differential entropy can be upper bounded by moment. The most common one is

$$
h(X) \leq \log \left(\sqrt{2 \pi e \mathbb{E}\left[\|X\|^{2}\right]}\right)
$$

where the identity is achieved by Gaussian distribution with zero means. More generally, $d P_{X^{*}}=e^{-V(x)} d x$ maximizes $h(X)$ among all the distributions with same $\mathbb{E}[V(X)]$, which is the direct consequence of $D\left(d P_{X} \| e^{-V(x)}\right) \geq 0$.

Another important inequality for differential entropy is EPI as stated in (2.25). It provides a clear expression to bound the differential entropy of 
two distributions' convolution. EPI has an analogy with Brunn-Minkowski inequality, which is a fundamental result in convex geometry. They are both special cases of strengthened Young's inequality [26, Lemma 17.8.1]. Recently, a strengthened EPI for log-concave measures is build by Courtade et al. using OT theory [51]. By Caffarelli's contraction theorem (c.f. Theorem 22), the Brenier map between a log-concave measure and a Gaussian is 1Lipschitz, which gives an estimate for the deficit of EPI. In our proofs below, readers can also find that our estimate is basically based on the convexity of Brenier potential $\varphi$.

However, in some situations, we need the lower bound of entropy, for instance, in the problem of upper bounding the rate-distortion function [52] and, similarly, in our case of upper bounding the Sinkhorn distance. Generally, it is a harder problem and we cannot give a reverse bound with finite moments for a universal case like (4.1). A example is the Dirac function, whose differential entropy is always $-\infty$ but the moments can be finite.

Under certain regularity, entropy bound can be reversed. Recent work of Marsiglietti and Kostina [52] also shows the differential entropy of a logconcave random variable $X$ can be lower bounded in terms of the $\mathrm{p}$-th absolute moment of $X$,

$$
h(X) \geq \log \frac{2\|X-\mathbb{E}[X]\|_{p}}{\Gamma(p+1)^{\frac{1}{p}}},
$$

when $p \geq 1$. When $p=2$, we have

$$
h(X) \geq \frac{1}{2} \log (4 \operatorname{Var}[X]) .
$$

Similar results also hold for EPI. Bobkov and Madiman [53] proved that reversibility of EPI is impossible for the whole family of convex probability distributions, i.e. the distribution is $f(x)=V(x)^{-\beta}, V(x)$ is positive convex, $x \in \mathbb{R}^{n}, \beta \geq n$.

However, Bobkov and Madiman proved there is a reversed version of EPI for log-concave measures, like Milman's reverse Brunn-Minkowski inequality for convex bodies.

Theorem 16 (Bobkov and Madiman's reverse EPI). [54] If $X$ and $Y$ are independent and have log-concave densities, then there exist some linear volume preserving maps (see Definition A.5.4) $u_{i}: \mathcal{R}^{n} \rightarrow \mathcal{R}^{n}$,

$$
N(\widetilde{X}+\widetilde{Y}) \leq C(N(X)+N(Y)),
$$

where $C$ is an absolute constant, $\tilde{X}=u_{1}(X), \tilde{Y}=u_{2}(Y)$. 
Corollary 16.1. Let $X, Y: \Omega \rightarrow \mathbb{R}$ be two independent scalar-valued random variables with $\log$-concave densities. Then

$$
N(X+Y) \leq C(N(X)+N(Y))
$$

Proof. From Theorem 16, $u_{i}$ is linear volume preserving map and $\operatorname{det}\left(D u_{i}\right)=$ 1 , the result can only be identity map.

(4.2) and (4.3) are related. Specifically, (4.2) leads to an explicit constant $C=\pi e / 2$ for $(4.3)$.

However, it is possible to lower bound the entropy if we have the Fisher information, for example, in (2.19) and (3.3). An intuitive explanation is that the Fisher information describes the regularity of a measure. For example, the Stam defect $p(X):=\frac{1}{n} N(X) J(X)=1$ only if $P_{X}$ is the most regular measure, which is Gaussian.

\subsubsection{Connections with Stochastic Process}

There are many works study the connections between entropic OT and old problems in stochastic process and fluid dynamics. Such problems include Fokker-Planck equation, Schrödinger's problem, Ornstein-Uhlenbeck process, etc.

We also give a point of view from dynamics in Sections 2.3, 2.4 and 3.2. From Theorem 6, we know the Wasserstein geodesic between two random vectors on multidimensional Euclidean space is linear interpolation. Our construction of the dynamics in the proof in the theorem 13 may also be explained as a stochastic process. More connections may be studied in the future works. In this subsection, we start from the geometry of OT introduced by Felix Otto.

Let $M$ be a Riemannian manifold. $\mathcal{P}(M)$ equipped with Wasserstein distance of order 2 as metric is so-called Wasserstein space, written as $\mathcal{P}_{2}(M)$.

Let $\mu_{t}, \mu_{t}^{\prime}$ be two geodesics starting from the same point on Wasserstein space, i.e., $\mu_{t}, \mu_{t}^{\prime} \in \mathcal{P}(M)$ for $t \in[0,1], \mu_{0}=\mu_{0}^{\prime}=\mu$. Their tangent vectors at $\mu \in \mathcal{P}(M)$ can be written as

$$
\dot{\mu}=-\nabla \cdot\left(\mu v_{1}\right), \quad \dot{\mu}^{\prime}=-\nabla \cdot\left(\mu v_{2}\right)
$$

where $v_{1}, v_{2}$ are the velocity fields defined in Section 2.3. From this setting, 
we can define inner product of Wasserstein space

$$
\left\langle\dot{\mu}, \dot{\mu}^{\prime}\right\rangle_{\mu}=\int_{M}\left\langle v_{1}, v_{2}\right\rangle d \mu
$$

where $\langle\cdot, \cdot\rangle$ is Riemannian metric. The inner product naturally induces a norm of the infinitesimal variation $\dot{\mu}$,

$$
\|\dot{\mu}\|_{\mu}=\inf \left\{\int_{M}|v|^{2} d \mu ; \quad \dot{\mu}+\nabla \cdot(v \mu)=0\right\} .
$$

Then there is a nice representation formala for the second order Wasserstein distance $\mathcal{W}_{2}$ :

$$
\mathcal{W}_{2}^{2}\left(\mu_{0}, \mu_{1}\right)=\inf \int_{0}^{1}\left\|\dot{\mu}_{t}\right\|_{\mu_{t}}^{2} d t
$$

The geometry of Wasserstein space leads to the following theorem.

Theorem 17 (Gradient of differential entropy is the Laplace operator). [28, Example 15.4] For a given measure $\mu \in \mathcal{P}_{2}(\mathcal{X}), \mathcal{X}=\mathbb{R}^{n}$, its gradient on Wasserstein space can be written as

$$
\operatorname{grad}_{\mu} h=\Delta \mu \text {. }
$$

Proof. Let $\mu_{t}$ be a trajectory on $\mathcal{P}(\mathcal{X})$ and let $\rho(x, t)$ be its density. Then its differential entropy is defined by

$$
h\left(\mu_{t}\right):=\int_{\mathcal{X}} \rho \log \rho d x .
$$

We calculate its time derivative,

$$
\frac{d}{d t} h\left(\mu_{t}\right)=\int_{\mathcal{X}}\left(\dot{\rho} \log \rho+\rho \frac{\dot{\rho}}{\rho}\right) d x=\int_{\mathcal{X}}(\log \rho+1) \dot{\rho} d x .
$$

Let $\boldsymbol{v}$ be the vector field of $\mu_{t}$. From the continuity equation $\dot{\rho}=-\nabla \cdot(\boldsymbol{v} \rho)$, we have

$$
\int_{\mathcal{X}} \dot{\rho} d x=-\int_{\mathcal{X}} \nabla \cdot(\boldsymbol{v} \rho) d x=-\int_{\partial \mathcal{X}} \boldsymbol{v} \rho \cdot \boldsymbol{n} d S=0 .
$$

where $S:=\partial \mathcal{X}$ is the piecewise smooth boundary. $\boldsymbol{n}$ is the outward pointing 
unit normal of $S$. Thus,

$$
\frac{d}{d t} h\left(\mu_{t}\right)=\int_{\mathcal{X}} \log \rho \nabla \cdot(\boldsymbol{v} \rho) d x
$$

Also,

$$
\nabla \cdot(\rho \log \rho \boldsymbol{v})=\log \rho \nabla \cdot(\boldsymbol{v} \rho)+\langle\nabla \log \rho, \rho \boldsymbol{v}\rangle .
$$

Then, we have

$$
\begin{aligned}
\frac{d}{d t} h\left(\mu_{t}\right) & =\left\langle\operatorname{grad}_{\mu} h, \dot{\mu}_{t}\right\rangle_{\mu_{t}} \\
& =\int_{\mathcal{X}}\langle\nabla \log \rho, \boldsymbol{v}\rangle \rho d x
\end{aligned}
$$

where (4.7) is the definition of gradient. From the definition of inner product (4.5), we know that the vector field of the gradient of differential entropy is $\nabla \log \rho$. Using continuity equation, we have

$$
\operatorname{grad}_{\mu} h=-\nabla \cdot(\boldsymbol{v} \rho)=-\nabla \cdot(\nabla \log \rho \cdot \rho)=\Delta \mu .
$$

Therefore, the gradient is the infinitesimal variation $\Delta \mu$.

In stochastic process, the Laplacian operator is also a generator of a Wiener process. We next state this result in the name of heat kernel. Let $Y_{t}=X+$ $\sqrt{t} Z$, where $Z \sim \mathcal{N}\left(0, I_{n}\right)$. We define $g_{t}(y)$ as the density of $Y_{t}$, then

$$
g_{t}(y)=\int_{\mathcal{X}} f(x)(2 \pi t)^{-\frac{n}{2}} e^{-\frac{(y-x)^{\top}(y-x)}{2 t}} d x .
$$

Theorem 18 (Heat Kernel). The probability density function $g_{t}(y)$ satisfies the heat equation, i.e.,

$$
\frac{\partial}{\partial t} g_{t}(y)=\frac{1}{2} \Delta g_{t}(y)
$$

Hence, the independent Gaussian vector $Z$ is called a heat kernel.

Proof. We compute the above terms respectively. First,

$$
\begin{aligned}
& \frac{\partial}{\partial t} g_{t}(y) \\
= & \int_{\mathcal{X}} f(x) \frac{\partial}{\partial t}\left[(2 \pi t)^{-\frac{n}{2}} e^{-\frac{(y-x)^{\top}(y-x)}{2 t}}\right] d x \\
= & \int_{\mathcal{X}} f(x)\left[(2 \pi t)^{-\frac{n}{2}-1}\left(-\frac{n}{2} \cdot 2 \pi\right)+(2 \pi t)^{-\frac{n}{2}} \frac{(y-x)^{\top}(y-x)}{2 t^{2}}\right] e^{-\frac{(y-x)^{\top}(y-x)}{2 t}} d x .
\end{aligned}
$$


We also calculate

$$
\nabla g_{t}(y)=\int_{\mathcal{X}} f(x)(2 \pi t)^{-\frac{n}{2}} \frac{-(y-x)}{t} e^{-\frac{(y-x)^{\top}(y-x)}{2 t}} d x
$$

and

$$
\begin{aligned}
\Delta g_{t}(y) & =\int_{\mathcal{X}} f(x)(2 \pi t)^{-\frac{n}{2}} \nabla \cdot\left[\frac{(y-x)}{t} e^{\left.-\frac{-(y-x) 2^{\top}(y-x)}{2 t}\right]}\right] d x \\
& =\int_{\mathcal{X}} f(x)(2 \pi t)^{-\frac{n}{2}}\left[-\frac{n}{t}+\frac{(y-x)^{\top}(y-x)}{t^{2}}\right] e^{-\frac{(y-x)^{\top}(y-x)}{2 t}} d x \\
& =2 \frac{\partial}{\partial t} g_{t}(y) .
\end{aligned}
$$

Then we finish our proof.

(4.8) directly leads to de Bruijn's identity, which states $\frac{d}{d t} h(X+\sqrt{t} Z)=$ $\frac{1}{2} J(X+\sqrt{t} Z)$. Equivalently, it can also be stated in term of Ornstein-Uhlenbeck process as $\frac{d}{d t} D\left(Y_{t} \| Z\right)=-I\left(Y_{t} \| Z\right)$. The identity can further derive EPI. Meanwhile, the probability measure of $Y_{t}$ can be a reference measure for Schrödinger's problem and coincides with the quadratic entropic OT.

We then briefly introduce the Schrödinger's problem [55]. Schrödinger's problem studies the entropic interpolation between two fixed marginals. To be consistent with the notations in statistical physics, we write $D(P \| Q)$ as $H(P \mid Q)$ in the next part.

Given a reference path measure $R \in \mathcal{M}_{+}(\Omega)$, where $\Omega=C([0,1], \mathcal{X})$, we define a canonical process on $\omega$ as

$$
X_{t}(\omega)=\omega_{t} \in \mathcal{X}, \quad \forall \omega=\left(\omega_{s}\right)_{0 \leq s \leq 1} \in \Omega, t \in[0,1] .
$$

Then the joint measure $R_{01}$ of the initial position $X_{0}$ and final position $X_{1}$ can be written as,

$$
R_{01}=\left(X_{0} \times X_{1}\right)_{\#} R \in \mathcal{M}_{+}\left(\mathcal{X}^{2}\right) .
$$

We first state the dynamic version of Schrödinger's problem.

Definition 4.2.1 (Dynamic Schrödinger's problem). The dynamic Schrödinger's problem associated with a given reference path measure $R \in \mathcal{M}_{+}(\Omega)$ is the following entropy minimization problem,

$$
H(P \mid R) \rightarrow \min ; \quad \text { s.t. } \quad P \in \mathcal{P}(\Omega): P_{0}=\mu_{0}, P_{1}=\mu_{0}, \quad\left(\mathrm{~S}_{d y n}\right)
$$

where $\mu_{0}, \mu_{1} \in \mathcal{P}(\mathcal{X})$ are the prescribed initial and final marginals. 
Then the static Schrödinger's problem can be considered as a projection of the dynamic Schrödinger's problem.

Definition 4.2.2 (Schrödinger's problem). The (static) Schrödinger's problem associated with a given reference measure $R_{01} \in \mathcal{M}_{+}\left(\mathcal{X}^{2}\right)$ is the following entropy minimization problem,

$$
H\left(\pi \mid R_{01}\right) \rightarrow \min ; \quad \text { s.t. } \quad \pi \in \mathcal{P}\left(\mathcal{X}^{2}\right): \pi_{0}=\mu_{0}, \pi_{1}=\mu_{1},
$$

where $\pi_{0}:=\pi(\cdot, \mathcal{X})$ and $\pi_{1}:=\pi(\mathcal{X}, \cdot) . \mu_{0}, \mu_{1} \in \mathcal{P}(\mathcal{X})$ are the prescribed intial and final marginals.

The equivalence between $\left(\mathrm{S}_{d y n}\right)$ and $(\mathrm{S})$ can be found in [56] if $R$ and $R_{01}$ satisfies (4.9).

Similar to OT and regularized OT, Schrödinger's problem also admits a dual formulation.

Theorem 19 (The dual problem). [57] Let $B: \mathcal{X} \rightarrow[1, \infty)$ be a measurable function such that

$$
\int_{\mathcal{X}^{2}} e^{-B(x)-B(y)} R_{01}(d x d y)<\infty
$$

Let $C_{B}(\mathcal{X})$ be the set of all continuous functions $u: \mathcal{X} \rightarrow \mathbb{R}$ such that $\sup |u| / B<\infty$ and $\mathcal{P}_{B}(\mathcal{X}):=\left\{\mu \in \mathcal{P}(\mathcal{X}) ; \int_{\mathcal{X}} B d \mu<\infty\right\}$. Then the dual problem of Schrödinger's problem (S) is stated as follows,

$$
\int_{\mathcal{X}} \varphi d \mu_{0}+\int_{\mathcal{X}} \psi d \mu_{1}-\log \int_{\mathcal{X}^{2}} e^{\varphi \oplus \psi} d R_{01} \rightarrow \max ; \quad \varphi, \psi \in C_{B}(\mathcal{X})
$$

where $\varphi \oplus \psi:(x, y) \in \mathcal{X}^{2} \rightarrow \varphi(x)+\psi(y) \in \mathbb{R}$. The prescribed marginals are assumed to satisfy $\mu_{0}, \mu_{1} \in \mathcal{P}_{B}(\mathcal{X})$.

The duality gives that $\inf (\mathrm{S})=\sup (\mathrm{D}) \in(-\infty, \infty]$. Suppose that $(\hat{\varphi}, \hat{\psi})$ and $\hat{\pi}$ are the optimizers for (D) and (S), respectively, then

$$
d \hat{\pi} / d R_{01}=\exp (\hat{\varphi} \oplus \hat{\psi}) .
$$

Under assumptions in [58, Theorem 2.8] like $R_{0}=R_{1}=m$, we can further write (4.10) in another form,

$$
\hat{\pi}(d x d y)=f_{0}(x) g_{1}(y) R_{01}(d x d y)
$$


where $f_{0}=e^{\varphi}$ and $g_{1}=e^{\psi} . f_{0}$ and $g_{1}$ satisfy following marginal constraints,

$$
\begin{cases}f_{0}(x) \mathbb{E}_{R}\left[g_{1}\left(X_{1}\right) \mid X_{0}=x\right]=d \mu_{0} / d m(x), & m \text {-a.e. } \\ g_{1}(y) \mathbb{E}_{R}\left[f_{0}\left(X_{0}\right) \mid X_{1}=y\right]=d \mu_{1} / d m(y), & m \text {-a.e. }\end{cases}
$$

These two constraints resemble (2.4) and (2.5) in the dual problem of regularized OT. It also leads to a unique solution of $\left(\mathrm{S}_{d y n}\right)$,

$$
\hat{P}=f_{0}\left(X_{0}\right) g_{1}\left(X_{1}\right) R \in \mathcal{P}(\Omega) .
$$

Then we show that $(\mathrm{S})$ and $\left(\mathcal{P}_{\epsilon}\right)$ coincide in a special case. Let the marginals be the Lebesgue measures, i.e., $R_{0}(d x)=d x$ and $R_{1}(d y)=d y$. Let the reference path measure be a Brownian motion, then the projected reference measure becomes a Gaussian kernel,

$$
R_{01}^{\epsilon}(d x d y)=(2 \pi \epsilon)^{-\frac{n}{2}} e^{-\frac{\|x-y\|^{2}}{2 \epsilon}} d x d y .
$$

We can write the relative entropy as,

$$
\epsilon H\left(\pi \mid R_{01}^{\epsilon}\right)=\int_{\mathcal{X}^{2}} \frac{\|x-y\|^{2}}{2} d \pi+\epsilon \int_{\mathcal{X}^{2}} \log \frac{d \pi}{d x d y} d \pi+\epsilon \log (2 \pi \epsilon)^{\frac{n}{2}} .
$$

From (4.13), we can easily see that $\left(\mathcal{P}_{\epsilon}\right)$ and $(\mathrm{S})$ admit the same optimizer when $c(x, y)=\|x-y\|^{2} / 2$ and that $R$ is a reversible Brownian motion from (4.12). It is also easy to easy to see that $\left(f_{0}, g_{1}\right)$ in (4.11a) and (4.11b) has following relation with $(\varphi, \psi)$ in $(\mathrm{S})$ and $\left(\mathcal{P}_{\epsilon}\right)$,

$$
\left\{\begin{array}{l}
a \cdot f_{0}(x)=e^{\varphi(x) / \epsilon} d \mu_{0} \\
b \cdot g_{1}(y)=e^{\psi(x) / \epsilon} d \mu_{1},
\end{array}\right.
$$

where $a, b \in \mathbb{R}_{+}$such that $a b=-(2 \pi \epsilon)^{\frac{n}{2}}$.

The equivalent dynamic formulation with a reference path measure of standard Brownian motion can be found in [58]. By simply re-scaling of the space $\mathcal{X}$, we can immediately get the follows from [58, Proposition 4.1],

$$
\begin{aligned}
& \inf \left\{H\left(\pi \mid R_{01}^{\epsilon}\right) ; \pi \in \mathcal{P}\left(\mathcal{X}^{2}\right): \pi_{0}=\mu_{0}, \pi_{1}=\mu_{1}\right\}-H\left(\mu_{0} \mid m\right) \\
= & \inf \left\{\int_{0}^{1} \frac{1}{2 \epsilon} \mathbb{E}_{\mu_{t}}\left[\|v\|^{2}\right] d t ;\left(\mu_{t}, v\right) \in \mathcal{V}\right\},
\end{aligned}
$$

where $\mathcal{V}$ is defined as the set of all $\left(\mu_{t}, v\right)$ such that have prescribed marginals 
$\mu_{0}, \mu_{1}$ and satisfy the Fokker-Planck equation,

$$
\dot{\mu}_{t}+\nabla \cdot\left(v \mu_{t}\right)=\frac{\epsilon}{2} \Delta \mu_{t},
$$

which corresponds to a stochastic differential equation (SDE),

$$
d X_{t}=v\left(X_{t}, t\right) d t+\sqrt{\epsilon} d W_{t}
$$

where $W_{t}$ is a standard Wiener process.

Thus, the regularized 2-Wasserstein distance admits a Benamou-Brenier formulation,

$$
\mathcal{W}_{d^{2}, \epsilon}\left(\mu_{0}, \mu_{1}\right)=\inf _{\left(\mu_{t}, v\right) \in \mathcal{V}} \int_{0}^{1} \mathbb{E}_{\mu_{t}}\left[\|v\|^{2}\right] d t / 2-\epsilon h\left(\mu_{1}\right)+\epsilon \log (2 \pi \epsilon)^{\frac{n}{2}}
$$

Therefore, the process is a Wassertein geodesic combined with a Brownian motion in the opposite direction of gradient of the differential entropy. Our construction of linear combination of independent random vectors in main results is actually an approximation of the continuous infinitesimal diffusion on time in (4.16). References for the connections between Schrödinger's problem, OT and functional inequality would be $[58,59,60]$.

From our discussion in Remark 3.1, we can see the EPI can be almost saturated on both sides of small and large $R$ and $C^{*}\left(P_{Y}, R\right) \approx \sqrt{1-e^{-\frac{2}{n} R}}$. One question is that, aside from our intuitive construction of linear combination $Y=Y_{1}+Y_{2}$, can we get a better estimate for the entropy power for the stochastic process described by the Fokker-Planck equation (4.15)? 


\section{References}

[1] M. Talagrand, "Transportation cost for gaussian and other product measures," Geometric \& Functional Analysis GAFA, vol. 6, no. 3, pp. 587-600, 1996.

[2] D. Bakry, F. Bolley, and I. Gentil, "Dimension dependent hypercontractivity for gaussian kernels," Probability Theory and Related Fields, vol. 154, no. 3, pp. 845-874, 2012.

[3] D. Cordero-Erausquin, "Transport inequalities for log-concave measures, quantitative forms, and applications," Canadian Journal of Mathematics, vol. 69, no. 3, pp. 481-501, 2017.

[4] F. Bolley, I. Gentil, A. Guillin et al., "Dimensional improvements of the logarithmic sobolev, talagrand and brascamp-lieb inequalities," The Annals of Probability, vol. 46, no. 1, pp. 261-301, 2018.

[5] M. Raginsky and I. Sason, "Concentration of measure inequalities in information theory, communications and coding," Foundations and Trends in Communications and Information Theory; NOW Publishers: Boston, MA, USA, 2018.

[6] R. Zhang, C. Chen, C. Li, and L. Carin, "Policy optimization as wasserstein gradient flows," in International Conference on Machine Learning. PMLR, 2018, pp. 5737-5746.

[7] G. Montavon, K.-R. Müller, and M. Cuturi, "Wasserstein training of restricted boltzmann machines," in Proceedings of the 30th International Conference on Neural Information Processing Systems, 2016, pp. 37183726.

[8] M. Arjovsky, S. Chintala, and L. Bottou, "Wasserstein generative adversarial networks," in International conference on machine learning. PMLR, 2017, pp. 214-223. 
[9] P. Rigollet and J. Weed, "Uncoupled isotonic regression via minimum wasserstein deconvolution," Information and Inference: A Journal of the IMA, vol. 8, no. 4, pp. 691-717, 2019.

[10] M. Cuturi, "Sinkhorn distances: lightspeed computation of optimal transport." in NIPS, vol. 2, no. 3, 2013, p. 4.

[11] Y. Bai, X. Wu, and A. Özgür, "Information constrained optimal transport: From talagrand, to marton, to cover," in 2020 IEEE International Symposium on Information Theory (ISIT). IEEE, 2020, pp. 2210-2215.

[12] C. E. Shannon, "A mathematical theory of communication," The Bell system technical journal, vol. 27, no. 3, pp. 379-423, 1948.

[13] A. J. Stam, "Some inequalities satisfied by the quantities of information of fisher and shannon," Information and Control, vol. 2, no. 2, pp. 101$112,1959$.

[14] O. Rioul, "Information theoretic proofs of entropy power inequalities," IEEE Transactions on Information Theory, vol. 57, no. 1, pp. 33-55, 2010.

[15] J. Adler, A. Ringh, O. Öktem, and J. Karlsson, "Learning to solve inverse problems using wasserstein loss," arXiv preprint arXiv:1710.10898, 2017.

[16] B. B. Damodaran, B. Kellenberger, R. Flamary, D. Tuia, and N. Courty, "Deepjdot: Deep joint distribution optimal transport for unsupervised domain adaptation," in Proceedings of the European Conference on Computer Vision (ECCV), 2018, pp. 447-463.

[17] Y. Wu and S. Verdú, "Witsenhausen's counterexample: A view from optimal transport theory," in 2011 50th IEEE Conference on Decision and Control and European Control Conference. IEEE, 2011, pp. 57325737.

[18] R. Gao and A. J. Kleywegt, "Distributionally robust stochastic optimization with wasserstein distance," arXiv preprint arXiv:1604.02199, 2016.

[19] I. Yang, "A convex optimization approach to distributionally robust markov decision processes with wasserstein distance," IEEE control systems letters, vol. 1, no. 1, pp. 164-169, 2017. 
[20] C. Zhao and Y. Guan, "Data-driven risk-averse stochastic optimization with wasserstein metric," Operations Research Letters, vol. 46, no. 2, pp. 262-267, 2018.

[21] B. Acciaio, J. Backhoff-Veraguas, and R. Carmona, "Extended mean field control problems: stochastic maximum principle and transport perspective," SIAM journal on Control and Optimization, vol. 57, no. 6, pp. 3666-3693, 2019.

[22] I. Yang, "Wasserstein distributionally robust stochastic control: A datadriven approach," IEEE Transactions on Automatic Control, 2020.

[23] G. Monge, "Mémoire sur la théorie des déblais et des remblais," Histoire de l'Académie Royale des Sciences de Paris, 1781.

[24] L. V. Kantorovich, "On the translocation of masses," in Dokl. Akad. Nauk. USSR (NS), vol. 37, 1942, pp. 199-201.

[25] C. Villani, Topics in optimal transportation. American Mathematical Soc., 2003, no. 58.

[26] T. M. Cover, Elements of information theory. John Wiley \& Sons, 1999.

[27] P. Billingsley, Convergence of probability measures. John Wiley \& Sons, 2013.

[28] C. Villani, Optimal transport: old and new. Springer Science \& Business Media, 2008, vol. 338.

[29] P. Dupuis and R. S. Ellis, A Weak Convergence Approach to the Theory of Large Deviations, 1 st ed., ser. Wiley series in probability and statistics. Hoboken: Wiley-Interscience, 2011. ISBN 9780471076728

[30] A. Genevay, "Entropy-regularized optimal transport for machine learning," Ph.D. dissertation, Paris Sciences et Lettres, 2019.

[31] L. Chizat, "Unbalanced optimal transport: Models, numerical methods, applications," Ph.D. dissertation, PSL Research University, 2017.

[32] R. Sinkhorn, "A relationship between arbitrary positive matrices and doubly stochastic matrices," The annals of mathematical statistics, vol. 35 , no. 2, pp. 876-879, 1964. 
[33] R. Sinkhorn and P. Knopp, "Concerning nonnegative matrices and doubly stochastic matrices," Pacific Journal of Mathematics, vol. 21, no. 2, pp. 343-348, 1967.

[34] R. Sinkhorn, "Diagonal equivalence to matrices with prescribed row and column sums," The American Mathematical Monthly, vol. 74, no. 4, pp. 402-405, 1967.

[35] J. Franklin and J. Lorenz, "On the scaling of multidimensional matrices," Linear Algebra and its applications, vol. 114, pp. 717-735, 1989.

[36] Y. Nesterov and A. Nemirovskii, Interior-point polynomial algorithms in convex programming. SIAM, 1994.

[37] J.-D. Benamou and Y. Brenier, "A computational fluid mechanics solution to the monge-kantorovich mass transfer problem," Numerische Mathematik, vol. 84, no. 3, pp. 375-393, 2000.

[38] F. Otto and C. Villani, "Generalization of an inequality by talagrand and links with the logarithmic sobolev inequality," Journal of Functional Analysis, vol. 173, no. 2, pp. 361-400, 2000.

[39] F. Otto, "The geometry of dissipative evolution equations: the porous medium equation," 2001.

[40] G. Blower, "The gaussian isoperimetric inequality and transportation," Positivity, vol. 7, no. 3, pp. 203-224, 2003.

[41] D. Bakry, M. Ledoux et al., "A logarithmic sobolev form of the liyau parabolic inequality," Revista Matemática Iberoamericana, vol. 22, no. 2, pp. 683-702, 2006.

[42] D. Bakry, I. Gentil, and M. Ledoux, Analysis and geometry of Markov diffusion operators. Springer Science \& Business Media, 2013, vol. 348.

[43] L. A. Stefanski and R. J. Carroll, "Deconvolving kernel density estimators," Statistics, vol. 21, no. 2, pp. 169-184, 1990.

[44] J. Fan, "On the optimal rates of convergence for nonparametric deconvolution problems," The Annals of Statistics, pp. 1257-1272, 1991. 
[45] E. Masry, "Multivariate probability density deconvolution for stationary random processes," IEEE Transactions on Information Theory, vol. 37, no. 4, pp. 1105-1115, 1991.

[46] H. Janati, B. Muzellec, G. Peyré, and M. Cuturi, "Entropic optimal transport between unbalanced gaussian measures has a closed form," Advances in Neural Information Processing Systems, vol. 33, 2020.

[47] T. A. Courtade, "A strong entropy power inequality," IEEE Transactions on Information Theory, vol. 64, no. 4, pp. 2173-2192, 2017.

[48] R. Flamary, N. Courty, A. Gramfort, M. Z. Alaya, A. Boisbunon, S. Chambon, L. Chapel, A. Corenflos, K. Fatras, N. Fournier, L. Gautheron, N. T. Gayraud, H. Janati, A. Rakotomamonjy, I. Redko, A. Rolet, A. Schutz, V. Seguy, D. J. Sutherland, R. Tavenard, A. Tong, and T. Vayer, "Pot: Python optimal transport," Journal of Machine Learning Research, vol. 22, no. 78, pp. 1-8, 2021. [Online]. Available: http://jmlr.org/papers/v22/20-451.html

[49] Y. Bengio, N. Le Roux, P. Vincent, O. Delalleau, and P. Marcotte, "Convex neural networks," Advances in neural information processing systems, vol. 18, p. 123, 2006.

[50] F. Bach, "Breaking the curse of dimensionality with convex neural networks," The Journal of Machine Learning Research, vol. 18, no. 1, pp. 629-681, 2017.

[51] T. A. Courtade, M. Fathi, and A. Pananjady, "Quantitative stability of the entropy power inequality," IEEE Transactions on Information Theory, vol. 64, no. 8, pp. 5691-5703, 2018. doi: 10.1109/TIT.2018.2808161

[52] A. Marsiglietti and V. Kostina, "A lower bound on the differential entropy of log-concave random vectors with applications," Entropy, vol. 20, no. 3, p. 185, 2018.

[53] S. G. Bobkov and M. M. Madiman, "On the problem of reversibility of the entropy power inequality," in Limit theorems in probability, statistics and number theory. Springer, 2013, pp. 61-74.

[54] S. Bobkov and M. Madiman, "Reverse brunn-minkowski and reverse entropy power inequalities for convex measures," Journal of Functional Analysis, vol. 262, no. 7, pp. 3309-3339, 2012. 
52|REFERENCES

[55] E. Schrödinger, Über die umkehrung der naturgesetze. Verlag der Akademie der Wissenschaften in Kommission bei Walter De Gruyter u ..., 1931 .

[56] H. Föllmer, "Random fields and diffusion processes," in École d'Été de Probabilités de Saint-Flour XV-XVII, 1985-87. Springer, 1988, pp. 101-203.

[57] C. Léonard, "Minimization of energy functionals applied to some inverse problems," Applied mathematics and optimization, vol. 44, no. 3, pp. 273-297, 2001.

[58] — _ "A survey of the schrödinger problem and some of its connections with optimal transport," Discrete \& Continuous Dynamical Systems, vol. 34, no. 4, pp. 1533-1574, 2014.

[59] Y. Chen, T. T. Georgiou, and M. Pavon, "On the relation between optimal transport and schrödinger bridges: A stochastic control viewpoint," Journal of Optimization Theory and Applications, vol. 169, no. 2, pp. 671-691, 2016.

[60] L. Ripani, "Le problème de Schrödinger et ses liens avec le transport optimal et les inégalités fonctionnelles," Theses, Université de Lyon, Dec. 2017. [Online]. Available: https://tel.archives-ouvertes.fr/ tel-01718846 


\section{Appendix A}

\section{More Proofs for Chapter 3}

\section{A.1 Proof of Theorem 12}

For a $C^{2}$ continuous function $V: \mathbb{R}^{n} \rightarrow \mathbb{R}, D^{2} V \geq \lambda I_{n}$, by Taylor formula [4, Lemma 2.5], there exists a $t \in[0,1]$ satisfying

$$
\begin{aligned}
& V(y)-V(x) \\
= & \nabla V(x) \cdot(y-x)+(y-x) \cdot D^{2} V(t x+(1-t) y)(y-x) / 2 \\
\geq & \nabla V(x) \cdot(y-x)+\frac{\lambda}{2}\|y-x\|^{2} .
\end{aligned}
$$

Hence we can bound the second order cost by

$$
\frac{\lambda}{2} \int_{\mathcal{X} \times \mathcal{Y}}\|y-x\|^{2} d P \leq \int_{\mathcal{X} \times \mathcal{Y}} V(y)-V(x)-\nabla V(x) \cdot(y-x) d P .
$$

Because entropic OT is a minimization problem, we can take any case in $\Pi\left(P_{X}, P_{Y} ; R\right)$ to bound $\mathcal{W}_{2}\left(P_{X}, P_{Y} ; R\right)$. We take a linear combination $Y=$ $Y_{1}+Y_{2}$, where $Y_{1}$ and $Y_{2}$ are independent, $h(Y)-h\left(Y_{2}\right) \leq R$ and $\mathbb{E}\left[Y_{2}\right]=0$. Assume there is a Brenier map between $Y_{1}$ and $X$, i.e., $Y_{1}=\nabla \varphi(X)$, which always exists, according to Theorem 2 (see Appendix A.5). Then, we can see this special case is in $\Pi\left(P_{X}, P_{Y}, R\right)$, namely,

$$
\begin{aligned}
I_{P}(X ; Y) & =h(Y)-h(Y \mid X) \\
& =h(Y)-h\left(Y_{1}+Y_{2} \mid X\right) \\
& =h(Y)-h\left(Y_{2}\right) \\
& \leq R .
\end{aligned}
$$


Let $d \mu=e^{-V}$, where $V: \mathbb{R}^{n} \rightarrow \mathbb{R}$ is $C^{2}$ continuous, $D^{2} V \geq \lambda I_{n}$. In order to bound Sinkhorn distance, we just need to bound $\int_{\mathcal{X} \times \mathcal{Y}} \nabla V(x) \cdot(y-x) d P$, according to (A.2). This term can be bounded as follows,

$$
\begin{aligned}
& \int_{\mathcal{X} \times \mathcal{Y}} \nabla V(x) \cdot(y-x) d P \\
= & \iint \nabla V(x) \cdot\left(\nabla \varphi(x)+y_{2}-x\right) d P_{Y_{2}} d P_{X} \\
= & \int \nabla V(x) \cdot(\nabla \varphi(x)-x) d P_{X} \\
= & \int \nabla V(x) \cdot(\nabla \varphi(x)-x) \frac{d P_{X}}{d \mu} d \mu \\
\geq & \int \Delta \varphi(x) f d \mu-n+\int(\nabla \varphi(x)-x) \cdot \nabla f d \mu \\
\geq & n e^{\frac{1}{n}\left(h\left(Y_{1}\right)-h(X)\right)}-n-\mathcal{W}_{2}\left(P_{X}, P_{Y_{1}}\right) \cdot \sqrt{I\left(P_{X} \mid \mu\right)},
\end{aligned}
$$

where we take the Radon-Nikodym derivative $f=\frac{d P_{X}}{d \mu}$ in (A.3) and apply Lemma 20 in Appendix A.5. This completes the derivation.

\section{A.2 Proof of Corollary $\mathbf{1 3 . 2}$}

Let $\mu_{A}=\frac{1_{A}}{\mu(A)} \mu$ and $\mu_{A_{r}}=\frac{1_{A_{r}}}{\mu(A)} \mu$ be the conditional probability measure $\mu$ restricted to $A$ and $A_{r}$. Using triangle inequality of $\mathcal{W}_{2}$, we have

$$
r \leq \mathcal{W}_{2}\left(\mu_{A}, \mu_{A_{r}} ; R\right) \leq \mathcal{W}_{2}\left(\mu_{A}, \mu\right)+\mathcal{W}_{2}\left(\mu, \mu_{A_{r}} ; R\right)
$$

From (2.15), we know

$$
\mathcal{W}_{2}\left(\mu_{A}, \mu\right) \leq \sqrt{2 \lambda^{-1} D\left(\mu_{A} \| \mu\right)}=\sqrt{2 \lambda^{-1} \log (1 / \mu(A))}:=c_{A} .
$$

Subsitute $c_{A}$ into (A.4) and use (3.2), then we have

$$
\begin{aligned}
& \left(r-c_{A}\right)^{2} \\
\leq & \mathcal{W}_{2}^{2}\left(\mu, \mu_{A_{r}} ; R\right) \\
\leq & \frac{2}{\lambda}\left(\mathbb{E}[V(Y)]-\mathbb{E}[V(X)]+n-n C e^{\frac{1}{n}(h(Y)-h(X))}\right) \\
= & \frac{2}{\lambda}\left(\mathbb{E}[V(Y)]-\mathbb{E}[V(X)]+n-n C e^{\frac{1}{n}\left(\mathbb{E}[V(Y)]-\mathbb{E}[V(X)]-D\left(\mu_{A_{r}} \| \mu\right)\right)}\right) .
\end{aligned}
$$


By (2.15), again we get, for $r \geq c_{A}$

$$
\mu\left(A_{r}\right) \leq C^{-n} \cdot e^{\mathbb{E}[V(X)]-\mathbb{E}[V(Y)]}\left[1+\frac{1}{n}\left(\mathbb{E}[V(Y)]-\mathbb{E}[V(X)]-\frac{\lambda}{2}\left(r-c_{A}\right)^{2}\right)\right]^{n} .
$$

(A.5) already indicates a concentration of measure. Since $(1+u / n)^{n} \leq e^{u}$, it can be further shown that (A.5) also implies normal concentration, as follows,

$$
\mu\left(A_{r}\right) \leq C^{-n} \cdot e^{-\frac{\lambda}{2}\left(r-c_{A}\right)^{2}} .
$$

Then we prove the decreasing of $C^{n}$ when the dimension increases. We assume that $C$ is a function non-decreasing with respect to $\frac{R}{n}$, since the saturation of EPI is related to entropy power and entropy power is defined by $\frac{1}{2 \pi e} e^{\frac{2}{n} h(X)}$. We take the logarithm of $C^{n}$ and compute the derivative with respect to $n$,

$$
\begin{aligned}
\frac{\partial}{\partial n} \log C\left(\frac{R}{n}\right) & =\log C\left(\frac{R}{n}\right)+\frac{n}{C} \frac{\partial}{\partial n} C\left(\frac{R}{n}\right) \\
& =\log C\left(\frac{R}{n}\right)+\frac{n C^{\prime}}{C} \cdot\left(-\frac{R}{n^{2}}\right) .
\end{aligned}
$$

We know that $C \in[0,1]$ and non-decreasing. Thus, (A.6) is less than 0 . We finish the proof of the decreasing of $C^{n}$ with respect to $n$.

\section{A.3 Proof of Theorem 14}

Let $X^{\prime}$ be a copy of $X . X$ can be written as a linear combination $X=t X^{\prime}+$ $X_{2}$, where $X_{2}$ is zero-mean and independent with $X^{\prime}, h(X)-h\left(X_{2}\right) \leq R$, $t \in[0,1]$. Assume there exists a Brenier map $Y=\nabla \varphi\left(X^{\prime}\right)$. Similar to the 
proof of Theorem 13, this case is also in $\Pi\left(P_{X}, P_{Y}, R\right)$. Then we have

$$
\begin{aligned}
& \int_{\mathcal{X} \times \mathcal{Y}} \nabla V(x) \cdot(y-x) d P \\
= & \iint \nabla V\left(t x^{\prime}+x_{2}\right) \cdot \nabla \varphi\left(x^{\prime}\right) d P_{X^{\prime}} d P_{X_{2}}-n \\
= & \iint\left(\nabla V\left(t x^{\prime}+x_{2}\right)-t \cdot \nabla V\left(x^{\prime}\right)\right) \cdot \nabla \varphi\left(x^{\prime}\right) d P_{X^{\prime}} d P_{X_{2}} \\
& +t \int \nabla V\left(x^{\prime}\right) \cdot \nabla \varphi\left(x^{\prime}\right) d P_{X^{\prime}}-n \\
= & \iint\left(\nabla V\left(t x^{\prime}+x_{2}\right)-t \cdot \nabla V\left(x^{\prime}\right)\right) \cdot \nabla \varphi\left(x^{\prime}\right) d P_{X^{\prime}} d P_{X_{2}} \\
& +t \int \Delta \varphi d P_{X^{\prime}}-n \\
\geq & t \cdot n e^{\frac{1}{n}(h(Y)-h(X))}-\epsilon-n,
\end{aligned}
$$

where we use Lemma 21 of Appendix A.5 in (A.7) and (A.8). In (A.9), we let $\epsilon=-\iint\left(\nabla V\left(t x^{\prime}+x_{2}\right)-t \cdot \nabla V\left(x^{\prime}\right)\right) \cdot \nabla \varphi\left(x^{\prime}\right) d P_{X^{\prime}} d P_{X_{2}}$ and apply (A.12). After changing the order of integral, we can see that $\int \nabla V\left(t x^{\prime}+x_{2}\right) d P_{X_{2}}$ is a smoothed version of $\nabla V\left(t x^{\prime}\right)$. When $\nabla V$ is a linear function perturbed by a zero mean noise, i.e., $\nabla V(t x)=t \cdot \nabla V(x)+W$, the integral of $x_{2}$ is cancelled out and $\epsilon=0$. Take $C_{x}\left(P_{X}, R\right)=t$, then we finish the proof.

\section{A.4 Proof of Theorem 15}

From Theorem 13, we know for any $z_{0}$, there exists such $P_{X, Y \mid Z=z_{0}} \in$ $\Pi\left(P_{X \mid Z=z_{0}}, P_{Y \mid Z=z_{0}} ; R\right)$ satisfying $I\left(X ; Y \mid Z=z_{0}\right) \leq R$ and follows,

$$
\begin{aligned}
& \frac{\lambda}{2} \mathbb{E}\left[\|X-Y\|^{2} \mid Z=z_{0}\right] \\
\leq & \mathbb{E}\left[V\left(Y \mid Z=z_{0}\right)\right]-\mathbb{E}[V(X)]+n-n C\left(P_{Y} \mid Z=z_{0}, R\right) e^{\frac{1}{n}\left(h\left(Y \mid Z=z_{0}\right)-h(X)\right)} \\
\leq & \mathbb{E}\left[V\left(Y \mid Z=z_{0}\right)\right]-\mathbb{E}[V(X)]+n-n C^{\prime}\left(P_{Y \mid Z}, R\right) e^{\frac{1}{n}\left(h\left(Y \mid Z=z_{0}\right)-h(X)\right)} .
\end{aligned}
$$

The conditional mutual information can be bounded in this case,

$$
I(X ; Y \mid Z)=\mathbb{E}_{P_{Z}}\left[I\left(X ; Y \mid Z=z_{0}\right)\right] \leq \mathbb{E}_{P_{Z}}[R]=R .
$$


Therefore, this $P_{X, Y \mid Z=z_{0}}$ yields the following estimate,

$$
\begin{aligned}
& \frac{\lambda}{2} \mathcal{W}_{2}^{2}\left(P_{X \mid Z}, P_{Y \mid Z} \mid P_{Z} ; R\right) \\
\leq & \frac{\lambda}{2} \mathbb{E}_{P_{Z}}\left\{\mathbb{E}\left[\|X-Y\|^{2} \mid Z=z_{0}\right]\right\} \\
\leq & \mathbb{E}[V(Y \mid Z)]-\mathbb{E}[V(X)]+n-n C^{\prime}\left(P_{Y \mid Z}, R\right) \mathbb{E}_{P_{Z}}\left[e^{\frac{1}{n}\left(h\left(Y \mid Z=z_{0}\right)-h(X)\right)}\right] \\
\leq & \mathbb{E}[V(Y \mid Z)]-\mathbb{E}[V(X)]+n-n C^{\prime}\left(P_{Y \mid Z}, R\right) e^{\frac{1}{n}\left(\mathbb{E}_{P_{Z}}\left[h\left(Y \mid Z=z_{0}\right)\right]-h(X)\right)} \\
= & \mathbb{E}[V(Y \mid Z)]-\mathbb{E}[V(X)]+n-n C^{\prime}\left(P_{Y \mid Z}, R\right) e^{\frac{1}{n}(h(Y \mid Z)-h(X))} .
\end{aligned}
$$

(A.10) follows from Jensen's inequality.

\section{A.5 Useful Definitions, Theorems and Lemmas}

Lemma 20. [25, Theorem 9.17] Let $d \mu=e^{-V}$. Let $f=\frac{d P_{X}}{d \mu}$ being a RadonNikodym derivative between two measures $P_{X}$ and $\mu$. Let $\nabla \varphi$ be a Brenier map as in Theorem 2. We have

$$
\begin{aligned}
& \int \nabla V(x) \cdot(\nabla \varphi(x)-x) f(x) d \mu(x) \\
\geq & \int[(\Delta \varphi-n) f+(\nabla \varphi-x) \cdot \nabla f] d \mu \\
= & \int \Delta \varphi f d \mu-n+\int(\nabla \varphi-x) \cdot \nabla f d \mu,
\end{aligned}
$$

where $\nabla \varphi(x)-x$ is called displacement. For the first term of (A.11), because $\varphi$ is convex, we have

$$
\int \Delta \varphi d P_{X} \geq n e^{\frac{1}{n}(h(\nabla \varphi(X))-h(X))} .
$$


Moreover, the last term of (A.11) can be bounded using Cauchy-Schwarz inequality as follows,

$$
\begin{aligned}
& \int(\nabla \varphi-x) \cdot \nabla f d \mu \\
\geq & -\left[\int\|\nabla \varphi-x\|^{2} f d \mu\right]^{1 / 2}\left[\int \frac{\|\nabla f\|^{2}}{f} d \mu\right]^{1 / 2} \\
= & -\mathcal{W}_{2}\left(P_{X}, \nabla \varphi_{\#} P_{X}\right) \cdot \sqrt{I\left(P_{X} \mid \mu\right)} .
\end{aligned}
$$

Lemma 21. [3, Fact 7] For any $\nabla \varphi \in L^{1}(\mathcal{X}) \cap L^{2}(\mathcal{X})$ on a Polish space $(\mathcal{X}, \mu)$ and $d \mu=e^{-V}$, we have

$$
\int \Delta \varphi d \mu=\int \nabla \varphi \cdot \nabla V d \mu
$$

Theorem 22 (Caffarelli). Let $\mu=e^{V}$ be a probability measure on $\mathbb{R}^{n}, V \in$ $C^{2}, D^{2} V>I_{d}$. Let $\gamma$ be the standard Gaussian measure. $T=\nabla \varphi$ is the Brenier map from $\gamma$ to $\mu$, i.e. $T_{\#} \gamma=\mu$. Then

$$
0 \leq D^{2} \varphi \leq I_{n}
$$

Caffarelli's log-concave perturbation theorem points out that the Brenier map between a Gaussian measure $\gamma$ and a "more log-concave" measure than $\gamma$ is 1Lipschitz. All the eigenvalues of $D^{2} \varphi$ are bounded between 0 and 1 . Through affine map, this can be generalized to common strong log-concave case.

Definition A.5.1 (Lower Semi-Continuity). Given a metric space $\mathcal{X}, f$ : $\mathcal{X} \rightarrow \mathbb{R} \cup\{\infty\}$ is lower semi-continuous if there exists a convergent sequence $\left\{x_{n}\right\}, x_{n} \rightarrow x$, that $f(x) \leq \liminf _{n \rightarrow \infty} f\left(x_{n}\right)$, where $\liminf _{n \rightarrow \infty} x_{n}:=\lim _{n \rightarrow \infty}\left(\inf _{m \geq n} x_{m}\right)$.

Definition A.5.2 (Log-Concavity). A density function $f$ with respect to Lebesgue measure on $\left(\mathbb{R}^{n}, \mathcal{B}^{n}\right)$ is log-concave if $f=e^{-V}$ where $V$ is a convex function.

Definition A.5.3 (Strong Log-Concavity). A density function $f$ is called strong log-concave if it has the form

$$
f(x)=g(x) \varphi(x)
$$

with some log-concave $g$ and some $\varphi \sim N(\mu, \Sigma)$.

Definition A.5.4 (Volume Preserving Maps). A diffeomorphism $u: \Omega \rightarrow \Omega$ is volume preserving if it satisfies any one of the following equivalent conditions: 
- $(u)_{\#} P=P$, which means $u$ pushes forward a measure $P$ to itself.

- $\operatorname{det}(D u)=1$.

Definition A.5.5 (Stochastic Kernel). Let $(\mathcal{X}, \mathcal{A})$ be a measurable space and $\mathcal{Y}$ a Polish space. Let $\tau(d y \mid x)$ be a family of probability measures on $\mathcal{Y}$ parametrized by $x \in \mathcal{X}$. We call $\tau(d y \mid x)$ a stochastic kernel on $\mathcal{Y}$ given $\mathcal{X}$ if for every Borel subset $E \subset \mathcal{Y}$, the mapping $x \in \mathcal{X} \rightarrow \tau(E \mid x) \in[0,1]$ is measurable.

Definition A.5.6 (Cyclical Monotonicity). [28, Definition 5.1] Let $\mathcal{X}, \mathcal{Y}$ be sets, and $c: \mathcal{X} \times \mathcal{Y} \rightarrow(-\infty,+\infty]$. A subset $\Gamma$ is said to be $c$-cyclical monotone if for any $N \in \mathbb{N}$ and any family $\left(x_{1}, y_{1}\right),\left(x_{2}, y_{2}\right), \ldots,\left(x_{N}, y_{N}\right)$ of points in $\Gamma$, holds the inequality,

$$
\sum_{i=1}^{N} c\left(x_{i}, y_{i}\right) \leq \sum_{i=1}^{N} c\left(x_{i}, y_{i+1}\right) .
$$

with the convention $y_{N+1}=y_{1}$. A transport plan is said to be $c$-cyclical monotone if it is concentrated on a $c$-cyclical monotone set.

Definition A.5.7 (c-convexity). [28, Definition 5.2] Let $\mathcal{X}, \mathcal{Y}$ be sets, and $c$ : $\mathcal{X} \times \mathcal{Y} \rightarrow(-\infty,+\infty]$. Then a function $\varphi: \mathcal{X} \rightarrow \mathbb{R} \cup\{+\infty\}$ is called $c$-convex if it is not identically $+\infty$, and there exists a function $\zeta: \mathcal{Y} \rightarrow \mathbb{R} \cap\{ \pm \infty\}$ such that

$$
\forall x \in \mathcal{X}, \quad \varphi(x)=\sup _{y \in \mathcal{Y}}(\zeta(y)-c(x, y)) .
$$

Its $c$-transform $\varphi^{c}$ is defined by

$$
\varphi^{c}(y)=\inf _{x \in \mathcal{X}}(\varphi(x)+c(x, y)) .
$$

The function $\varphi$ and $\varphi^{c}$ are said to be $c$-conjugate.

The $c$-subdifferential of $\varphi(x)$ is the $c$-cyclical monotone set defined by

$$
\partial_{c} \varphi:=\left\{(x, y) \in \mathcal{X} \times \mathcal{Y}: \varphi^{c}(y)-\varphi(x)=c(x, y)\right\} \text {. }
$$

Definition A.5.8 (c-concavity). [28, Definition 5.7] Let $\mathcal{X}, \mathcal{Y}$ be sets, and $c$ : $\mathcal{X} \times \mathcal{Y} \rightarrow(-\infty,+\infty]$. Then a function $\phi: \mathcal{Y} \rightarrow \mathbb{R} \cup\{-\infty\}$ is called $c$-convex if it is not identically $-\infty$, and there exists a function $\psi: \mathcal{Y} \rightarrow \mathbb{R} \cap\{ \pm \infty\}$ such that $\phi=\psi^{c}$. Its $c$-transform $\phi^{c}$ is defined by

$$
\phi^{c}(x)=\sup _{y \in \mathcal{Y}}(\phi(y)-c(x, y)) .
$$


60 | Appendix A: More Proofs for Chapter 3

The $c$-subdifferential of $\phi(x)$ is the $c$-cyclical monotone set defined by

$$
\partial_{c} \phi:=\left\{(x, y) \in \mathcal{X} \times \mathcal{Y}: \phi(y)-\phi^{c}(x)=c(x, y)\right\} \text {. }
$$




\section{For DIVA}

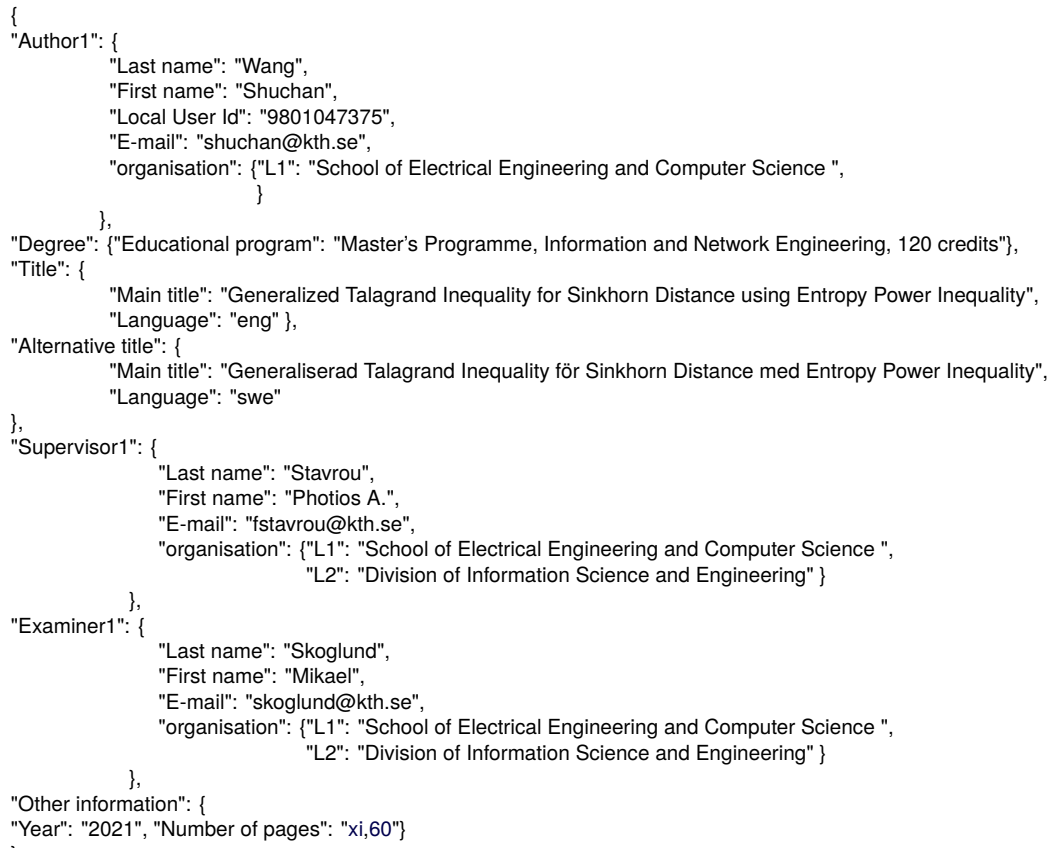


TRITA-EECS-EX-2021:893

www.kth.se 